\title{
Some approximate Godunov schemes to compute shallow-water equations with topography
}

\author{
Thierry Gallouët ${ }^{1}$, Jean-Marc Hérard ${ }^{1,2}$, Nicolas Seguin ${ }^{1,2}$ \\ ${ }^{1}$ Laboratoire d'Analyse Topologie et Probabilités - UMR 6632 \\ Centre de Mathématique et Informatique \\ Université de Provence \\ 39 rue Joliot Curie \\ 13453 MARSEILLE CEDEX 13 \\ ${ }^{2}$ Département Mécanique des Fluides et Transferts Thermiques \\ Électricité de France - Recherche et Développement \\ 6 quai Watier \\ 78401 CHATOU CEDEX
}

\begin{abstract}
We study here the computation of shallow-water equations with topography by Finite Volume methods, in a one-dimensional framework (though all methods introduced may be naturally extended in two dimensions). All methods performed are based on a dicretisation of the topography by a piecewise function constant on each cell of the mesh, from an original idea of A.Y. Le Roux et al.. Whereas the Well-Balanced scheme of A.Y. Le Roux is based on the exact resolution of each Riemann problem, we consider here approximate Riemann solvers, namely the VFRoencv schemes. Several single step methods are derived from this formalism, and numerical results are compared to a fractional step method. Some tests cases are presented : convergence to steady states in subcritical and supercritical configurations, occurence of dry area by a drain over a bump and occurence of vacuum by a double rarefaction wave over a step. Numerical schemes, combined with an appropriate high order extension, provide accurate and convergent approximations.
\end{abstract}




\section{Contents}

1 Introduction $\quad 4$

2 The shallow-water equations with topography $\quad 5$

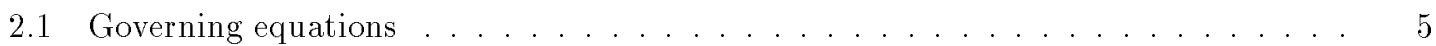

2.2 The Riemann problem on a flat bottom . . . . . . . . . . . . . . . 6

2.3 The Riemann problem with a piecewise constant topography . . . . . . . . . . 7

3 Single step methods $\quad 9$

3.1 An approximate Godunov-type scheme . . . . . . . . . . . . . . . . . . . . . 9

3.2 The VFRoencv formalism . . . . . . . . . . . . . . . . . . . . . . . 10

3.3 The VFRoe $\left(Z_{f}, h, Q\right)$ scheme . . . . . . . . . . . . . . . . 11

3.4 The VFRoencv $\left(Z_{f}, 2 c, u\right)$ scheme . . . . . . . . . . . . . . . . . . 12

3.5 The VFRoencv $\left(Z_{f}, Q, \psi\right)$ scheme . . . . . . . . . . . . . . 13

4 Fractional step method $\quad 15$

4.1 The VFRoencv $(2 c, u)$ scheme . . . . . . . . . . . . . . . . 15

4.2 The fractional step method . . . . . . . . . . . . . . . . . 16

$\begin{array}{llr}5 & \text { A higher order extension } & 17\end{array}$

$\begin{array}{llr}6 & \text { Numerical results } & 20\end{array}$

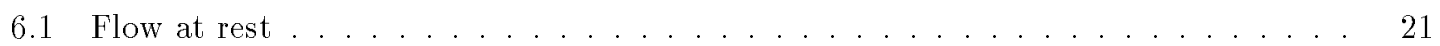

6.2 Subcritical flow over a bump . . . . . . . . . . . . . . . . . . . . 22

6.3 Transcritical flow over a bump . . . . . . . . . . . . . . . . . . . . . 22

6.4 Drain on a non flat bottom ...................... 24

6.5 Vacuum occurence by a double rarefaction wave over a step . . . . . . . . . 25

7 Conclusion $\quad 26$ 
A Comparison with the Well-Balanced scheme

A.1 Subcritical flow over a bump . . . . . . . . . . . . . . . . . 27

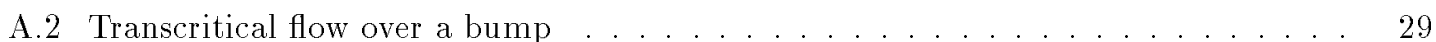

B Comparison with the VFRoe $\left(Z_{f}, h, Q\right)$ scheme 


\section{Introduction}

We study in this paper some approximate Godunov schemes to compute shallow-water equations with a source term of topography, in a one-dimensional framework. All methods presented may be extended naturally to the $2 \mathrm{D}$ model.

Shallow-water equations are based on conservation laws and provide an hyperbolic system. However, topography introduces some source term related to the unknown. Hence, analytic properties of the model of isentropic Euler equations are deeply modified, in comparison with the homogeneous case. For instance, a well-known problem is the occurence of other equilibrium states (or steady states), due to the presence of the source term.

Several ways to compute conservation laws with source term have already been investigated. The main problem is the approximation of the source term and the numerical preservation of properties fulfilled by the continuous system. Some Finite Volume method have been proposed, in particular the Well Balanced schemes, which can maintain all steady states. These schemes have been initially introduced by J.M. Greenberg and A.Y. Le Roux in [16] and [17] in the scalar case (see also [14] and [2]). Well Balanced schemes have been recently extended to shallow-water equations with topography in [1] and [18] and friction in [6]. Since the Well Balanced scheme is based on an exact Riemann solver as the Godunov scheme (see [13]), its main drawbacks are its calculation cost and the need to compute the "exact" solution of the Riemann problem. Other Finite Volume methods to deal with source terms exist too, for instance based on the Roe scheme (see [23] and [12]), or based on another approximation of the source term, like in [20].

Some properties of the continuous model (Riemann invariants, jump relations, ...) are first exposed, and a study of the Riemann problem is briefly recalled. Thereafter, some Godunov schemes are introduced to compute shallow-water equations, derived from the VFRoencv formalism (see [5]). Some applications of VFRoencv schemes are provided for the Euler equations (in [22], [5] and [11]), for shallow-water equations with a flat bottom in [4] and for turbulent compressible flows [3]. The VFRoencv schemes are based on an arbitrary change of variable, and on a linearisation of each interface Riemann problem. In the homogeneous case, the numerical flux is defined using the exact solution of the linearised Riemann problem and the conservative flux. However, the source term "breaks" the conservativity of the model. Thus, using a piecewise constant function to approximate the bottom, some approximate Riemann solvers are presented. The main advantages of this approach are the natural integration of the source term in the numerical methods and the use of a linearised Riemann problem, which minimizes the CPU time. Note that a scheme which exactly preserves a large class of steady states is obtained. In addition, a fractionnal step method is performed, based on the VFRoencv scheme introduced in [4]. This method enables to deal with vacuum and provides good results too. To complete this presentation, a higher order extension is provided, to increase the accuracy of the schemes when computing unsteady configurations or flows at rest.

Several numerical experiments are presented. All the test cases are one-dimensional, and are based on a non trivial topography. Indeed, applications of shallow-water equations are one-dimensional or two-dimensional configurations. Hence, computational limitations are rather different from the gas dynamics and numerical experiments may be performed on mesh containing several hundreds 
nodes. The tests include subcritical and transcritical flows over a bump [15] and a drain with a non flat bottom. The convergence towards steady states is measured. A vacuum occurence by a double rarefaction wave over a step is tested too. All the numerical tests confirm the good behaviour of the numerical methods, including the fractional step method.

Eventually, some complementary tests with the Godunov and the VFRoe method are provided in appendix.

\section{The shallow-water equations with topography}

\subsection{Governing equations}

The shallow-water equations represent a free surface flow of incompressible water. The twodimensional system may be written as follows :

$$
\begin{aligned}
& h_{, t}+(h u)_{, x}+(h v)_{, y}=0 \\
& (h u)_{, t}+\left(h u^{2}\right)_{, x}+(h u v)_{, y}+g\left(\frac{h^{2}}{2}\right)_{, x}=-g h\left(Z_{f}\right)_{, x} \\
& (h v)_{, t}+(h u v)_{, x}+\left(h v^{2}\right)_{, y}+g\left(\frac{h^{2}}{2}\right)_{, y}=-g h\left(Z_{f}\right)_{, y}
\end{aligned}
$$

where $h$ denotes the water height, $\mathbf{u}={ }^{t}(u, v)$ the velocity, $g$ the gravity constant and $\nabla Z_{f}$ the bed slope $\left(g\right.$ and $Z_{f}(x, y)$ are given, and $Z_{f}$ must be at least $\left.\mathcal{C}^{0}\left(\mathbb{R}^{2}\right)\right)$ (see figure 1 ).

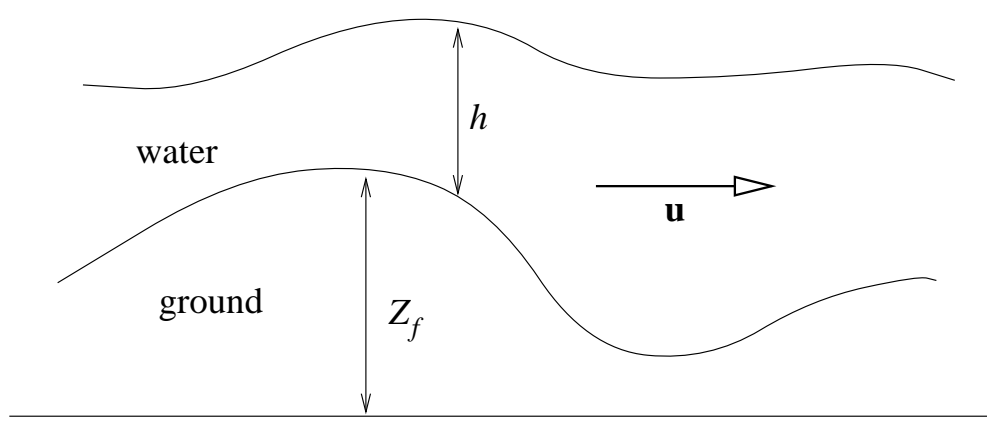

Figure 1: Mean variables

This study is restricted to the computation by Finite Volume schemes (see [9]). Since the hyperbolic system (1) remains unchanged under frame rotation, this two-dimensional problem may be solved 
considering on each interface of the mesh the following system :

$$
\begin{aligned}
& h_{, t}+\left(h u_{n}\right)_{, n}=0 \\
& \left(h u_{n}\right)_{, t}+\left(h u_{n}^{2}+g \frac{h^{2}}{2}\right)_{, n}=-g h\left(Z_{f}\right)_{, n} \\
& \left(h u_{\tau}\right)_{, t}+\left(h u_{n} u_{\tau}\right)_{, n}=0
\end{aligned}
$$

where $u_{n}=\mathbf{u} . n, u_{\tau}=\mathbf{u} . \tau, n$ and $\tau$ the normal and the tangential vector to the interface $(\|n\|=$ $\|\tau\|=1)$, and ()$, n$ the derivate along the normal vector $n$.

The pure one-dimensional shallow-water equations may be written as follows :

$$
\begin{aligned}
& h_{, t}+(h u)_{, x}=0 \\
& (h u)_{, t}+\left(h u^{2}+g \frac{h^{2}}{2}\right)_{, x}+g h Z_{f}^{\prime}(x)=0 .
\end{aligned}
$$

We focus in this paper on the numerical resolution of the one-dimensional system (3).

Let us note that $h$ and $h u$ (also denoted $Q$ in the following) are the conservative variables. So, vacuum (or dry bed) may be represented by $h=h u=0$, which implies that $u$ is not defined.

Remark 1. The change of variable from $(h, Q)$ to $(h, u)$ leads to the following equations for smooth solutions:

$$
\begin{aligned}
& h_{, t}+Q_{, x}=0 \\
& u_{, t}+\left(\frac{u^{2}}{2}+g\left(h+Z_{f}\right)\right)_{, x}=0 .
\end{aligned}
$$

where $\psi=\left(u^{2} / 2+g\left(h+Z_{f}\right)\right)$.

These equations enable to define some stationary smooth solutions as follows :

$$
Q_{, x}=0 \quad \text { and } \quad \psi_{, x}=0
$$

One may add to these equations Rankine Hugoniot relations (on smooth topography) for stationary shocks to complete the definition of stationary states.

\subsection{The Riemann problem on a flat bottom}

Assuming that the river bed is flat (ie $Z_{f}^{\prime}(x)=0$ ), the system (3) becomes homogeneous. Hence, we obtain a conservative system, which lead to the following Riemann problem :

$$
\left\{\begin{array}{l}
h_{, t}+Q_{, x}=0 \\
Q_{, t}+\left(\frac{Q^{2}}{h}+g \frac{h^{2}}{2}\right)_{, x}=0 \\
(h, Q)(x, 0)= \begin{cases}\left(h_{L}, Q_{L}\right) & \text { if } x<0, \\
\left(h_{R}, Q_{R}\right) & \text { if } x>0 .\end{cases}
\end{array}\right.
$$


This problem, which is also the Riemann problem for isentropic Euler equations (for a particular state law) may be classically solved. Its solution is a similarity solution (ie a function of $x / t$ ) composed by three constant states, $\left(h_{L}, Q_{L}\right),\left(h_{1}, Q_{1}\right)$ and $\left(h_{R}, Q_{R}\right)$ separated by two Genuinely Non Linear fields associated eith eigenvalues $u-c$ and $u+c$ (where $c=\sqrt{g h}$ ). The intermediate state $\left(h_{1}, Q_{1}\right)$ may be computed using through the 1 -wave :

$$
u_{1}= \begin{cases}u_{L}-2\left(\sqrt{g h_{1}}-\sqrt{g h_{L}}\right) & \text { if } h_{1}<h_{L}, \\ u_{L}-\left(h_{1}-h_{L}\right) \sqrt{g \frac{h_{1}+h_{L}}{2 h_{1} h_{L}}} & \text { if } h_{1}>h_{L} .\end{cases}
$$

and through the 2-wave :

$$
u_{1}= \begin{cases}u_{R}+2\left(\sqrt{g h_{1}}-\sqrt{g h_{R}}\right) & \text { if } h_{1}<h_{R}, \\ u_{R}+\left(h_{1}-h_{R}\right) \sqrt{g \frac{h_{1}+h_{R}}{2 h_{1} h_{R}}} & \text { if } h_{1}>h_{R} .\end{cases}
$$

The latter two curves are derived from the Riemann invariants (when $h_{1}<h_{L}$ and $h_{1}<h_{R}$ ) for rarefaction waves and from the Rankine Hugoniot relations (when $h_{1}>h_{L}$ and $h_{1}>h_{R}$ ) for shock waves. Note that the intermediate velocity $u_{1}$ is defined only if :

$$
u_{R}-u_{L}<2\left(\sqrt{g h_{R}}+\sqrt{g h_{L}}\right)
$$

Otherwise, $h_{1}$ and $Q_{1}$ become null, and $u_{1}$ is undefined.

\subsection{The Riemann problem with a piecewise constant topography}

Following the idea developed by A.Y. Le Roux in [18], the topography is described by a piecewise constant function. Therefore, adding the "partial" differential equation concerning $Z_{f}$, the following Riemann problem may be obtained :

$$
\left\{\begin{array}{l}
Z_{f, t}=0 \\
h_{, t}+(h u)_{, x}=0 \\
Q_{, t}+\left(\frac{Q^{2}}{h}+g \frac{h^{2}}{2}\right)_{, x}+g h\left(Z_{f}\right)_{, x}=0 \\
\left(h, Q, Z_{f}\right)(x, 0)= \begin{cases}\left(h_{L}, Q_{L}, Z_{f_{L}}\right) & \text { if } x<0, \\
\left(h_{R}, Q_{R}, Z_{f_{R}}\right) & \text { if } x>0 .\end{cases}
\end{array}\right.
$$

Note that this Riemann problem does not correspond to the Riemann problem associated with the system (3), since the topography is not smooth. The jump of topography along the curve $x / t=0$ introduces a problem for the definition of the product of distributions, focusing on non smooth solutions (see [7] and [8] for more details). So, the jump relations across the discontinuity $x / t=0$ are not defined. Assuming that $h>0$ and restricting to smooth solutions, the system (9) may be written :

$$
\begin{aligned}
& Z_{f, t}=0 \\
& h_{, t}+Q_{, x}=0 \\
& u_{, t}+\left(\frac{u^{2}}{2}+g\left(h+Z_{f}\right)\right)_{, x}=0 .
\end{aligned}
$$


We note $\psi=\left(u^{2} / 2+g\left(h+Z_{f}\right)\right)$ in the following. One may deduce the conservation law on entropy for non viscous smooth solutions :

$$
\begin{gathered}
\eta_{, t}+(Q \psi)_{, x}=0 \\
\eta=h \frac{u^{2}}{2}+g \frac{h^{2}}{2}+g h Z_{f} .
\end{gathered}
$$

Moreover, system (10) provides the Riemann invariants through the stationary wave. Since the wave located at $x / t=0$ is a contact discontinuity, we assume that the Rankine Hugoniot relations identifies with the Riemann invariants. Thus, the Riemann problem (9) admits a Linearly Degenerated field of speed 0 such that :

$$
\begin{aligned}
& \llbracket Q \rrbracket=0 \\
& \llbracket \psi \rrbracket=0
\end{aligned}
$$

where $\llbracket \alpha \rrbracket$ represents the jump of $\alpha$ across the wave.

Two Genuinely Non Linear fields also compose the solution of the Riemann problem (9), which are the same as in the flat bottom case. Hence, to connect a state $W$ to a state $W_{a}$ through the wave $u-c$, one may use the following relations (a rarefaction wave occurs when $h<h_{a}$, and a shock wave occurs when $h>h_{a}$ ):

$$
\begin{aligned}
Z_{f} & =Z_{f_{a}} \\
u & = \begin{cases}u_{a}-2\left(\sqrt{g h}-\sqrt{g h_{a}}\right) & \text { if } h<h_{a}, \\
u_{a}-\left(h-h_{a}\right) \sqrt{g \frac{h+h_{a}}{2 h h_{a}}} & \text { if } h>h_{a} .\end{cases}
\end{aligned}
$$

In the same way, to connect a state $W$ to a state $W_{b}$ through the wave $u+c$, one may use the following relations (a rarefaction wave occurs when $h<h_{b}$, and a shock wave occurs when $h>h_{b}$ ):

$$
\begin{aligned}
Z_{f} & =Z_{f_{b}} \\
u & =\left\{\begin{array}{l}
u_{b}+2\left(\sqrt{g h}-\sqrt{g h_{b}}\right) \\
u_{b}+\left(h-h_{b}\right) \sqrt{g \frac{h+h_{b}}{2 h h_{b}}} \text { if } h>h_{b} .
\end{array}\right.
\end{aligned}
$$

Moreover, to connect a state $W$ to a state $W_{c}$ through the stationary wave, one uses the Riemann invariants :

$$
\begin{aligned}
Q & =Q_{c} \\
\psi & =\psi_{c}
\end{aligned}
$$

Note that the exact resolution of the Riemann problem (9) is not obvious, though all fields and associated Riemann invariants and jump relations are known. Indeed, contrary to a "classical" Riemann problem (Euler equations for instance), the three waves of this Riemann problem are not ordered and a GNL wave may be superposed with the LD wave (see [18]). 
We will discuss below two families of schemes which are intended to provide a convergent approximation of the above mentionned system. The first series is based on straightforward approximate Godunov schemes which account for topography. The second series is based on the fractional step method.

\section{Single step methods}

We present in this section several ways to solve the shallow-water equations with source term by Finite Volume schemes (see [9] and [24] for instance). The description of the methods computed herein is split in two steps : the Finite Volume scheme provided by integration of (3) and the solver at each interface.

\subsection{An approximate Godunov-type scheme}

We introduce herein a Finite Volume scheme following the idea proposed by J.M. Greenberg, A.Y. Le Roux et al in [16] and [17].

Focusing on system (3), it consists in using a piecewise bottom, flat on each cell, in the "continuous" framework (see [18] and [6]). Thus, the source term $-g h Z_{f}^{\prime}(x)$ is reduced to a sum of Dirac mass occuring on each interface [7]. Hence, since the Finite Volume formalism is based on the integration of the system (3) on a cell $] x_{i-1 / 2} ; x_{i+1 / 2}\left[\times\left[t^{n} ; t^{n+1}[\right.\right.$, the source term does not appear explicitly (contrary to the scheme investigated in [12] for instance). As mentionned above, such an approximation of the topography introduces a a stationary wave at the interface of each local Riemann problem. Though the Well Balanced scheme of J.M. Greenberg and A.Y. Le Roux is based on the exact solution of (9), we focus here on approximate Riemann solvers. These Riemann solvers are based on an approximate solution of the problem (9), and the numerical flux is computed from the conservative flux and the approximate solution at each interface.

Let us note $W={ }^{t}\left(Z_{f}, h, Q\right)$ the conservative variable, $F(W)={ }^{t}\left(0, Q, h u^{2}+g h^{2} / 2\right)$ the associated conservative flux and $\Delta x_{i}$ and $\Delta t$ the space and time steps. We denote $W_{i}^{n}$ the approximation de $\frac{1}{\Delta x_{i}} \int_{x_{i-1 / 2}}^{x_{i+1 / 2}} W\left(x, t^{n}\right) d x$.

So, the Finite Volume scheme may be written as follows :

$$
\begin{aligned}
W_{i}^{n+1}=W_{i}^{n}-\frac{\Delta t}{\Delta x_{i}} & \left(F\left(W_{i+1 / 2}^{*}\left(0^{-} ; W_{i}, W_{i+1}\right)\right)\right. \\
& \left.-F\left(W_{i-1 / 2}^{*}\left(0^{+} ; W_{i-1}, W_{i}\right)\right)\right)
\end{aligned}
$$

where $W_{i+1 / 2}^{*}\left(x / t ; W_{i}, W_{i+1}\right)$ is the (exact or approximate) solution of the Riemann problem (9) with $L=i$ and $R=i+1$. As mentionned above, the source term only contributes to the computation of the (exact or approximate) solutions $W_{i+1 / 2}^{*}\left(x / t ; W_{i}, W_{i+1}\right)$ but it does not appear explicitly in the expression of the scheme (17). However, the approximation of the topography by a piecewise constant fonction implies that the numerical flux is not continuous through each interface of the mesh, contrary to the homogenous and conservative case. So, whereas the numerical 
flux associated with equation (3a) has to be continuous (since this equation is homogenous and conservative), the numerical flux associated with equation ( $3 \mathrm{~b}$ ) becomes discontinuous in the non flat bottom case, according to the relations (13). In order to obtain a constant numerical flux for equation (3a), we will have, in some cases, to modify the scheme (17) (see (21) for instance).

Note that the Finite Volume scheme (17) associated with the exact interface Riemann solver (ie the Well-Balanced scheme presented in [18]) is able to maintain all steady states. Moreover, let us emphasize that the scheme (17) may be easily extended to a multi-dimensional framework (indeed, the formalism presented is very similar to Finite Volume schemes).

\subsection{The VFRoencv formalism}

Since the Well Balanced scheme ([18]) is based on an exact Riemann solver as the Godunov scheme ([13]), its main drawbacks are its calculation cost and the need to compute the exact solution of the Riemann problem (9). Thus, the state $W_{i+1 / 2}^{*}\left(x / t ; W_{i}, W_{i+1}\right)$ is computed by approximate Riemann solvers.

All the Riemann solvers presented here may be derived from the VFRoencv formalism [5, 11]. The VFRoencv schemes are based on the exact solution of a linearised Riemann problem. Their construction may be split in three steps. The first step consists in writting the initial system under a non-conservative form, by an arbitrary change of variable $Y(W)$ (we denote by $W(Y)$ the inverse change of variable). Afterwards, the Riemann problem (9) is linearised averaging the convection matrix :

$$
\left\{\begin{array}{l}
Y_{, t}+B(\hat{Y}) Y_{, x}=0 \\
Y(x, 0)= \begin{cases}Y_{L}=Y\left(W_{L}\right) & \text { if } x<0 \\
Y_{R}=Y\left(W_{R}\right) & \text { if } x>0\end{cases}
\end{array}\right.
$$

where $B(Y)=\left(W_{, Y}(Y)\right)^{-1} F_{, W}(W(Y)) W_{, Y}(Y)$ and $\hat{Y}=\frac{Y_{L}+Y_{R}}{2}$.

As a result, the Riemann problem (9) becomes a linear Riemann problem, which is solved exactly. Denoting $\left(\tilde{l_{k}}\right)_{k=1,2,3}$ and $\left(\widetilde{r_{k}}\right)_{k=1,2,3}$ respectively left and right eigenvectors of $B(\widehat{Y}),\left(\widetilde{\lambda_{k}}\right)_{k=1,2,3}$ eigenvalues of $B(\hat{Y})$, the exact solution $Y^{*}\left(x / t ; Y_{L}, Y_{R}\right)$ of $(18)$ is defined by :

$$
\begin{aligned}
Y^{*}\left(x / t ; Y_{L}, Y_{R}\right) & =Y_{L}+\sum_{x / t<\widetilde{\lambda_{k}}}\left({ }^{t} \widetilde{l_{k}} \cdot \llbracket Y \rrbracket_{L}^{R}\right) \widetilde{r_{k}} \\
& =Y_{R}-\sum_{x / t>\widetilde{\lambda_{k}}}\left(\widetilde{l_{k}} \cdot \llbracket Y \rrbracket_{L}^{R}\right) \widetilde{r_{k}}
\end{aligned}
$$

where $\llbracket \alpha \rrbracket_{L}^{R}=\alpha_{R}-\alpha_{R}$. Thus, the solution written related to the conservative variable is

$$
W^{*}\left(x / t ; W_{L}, W_{R}\right)=W\left(Y^{*}\left(x / t ; Y_{L}, Y_{R}\right)\right) .
$$

On a conservative and homogeneous framework, the numerical flux is defined by the conservative flux computed with the approximate solution at the interface $x / t=0$. However, the Riemann 
problem (9) provides a stationary wave at the interface, which introduces a jump of the numerical flux across it (which appears even when the exact solution of (9) is computed).

We emphasize that the source term of topography $-g Z_{f}^{\prime}(x)$ appears naturally and explicitly in the expression of intermediate states computed by the following schemes.

\subsection{The VFRoe $\left(Z_{f}, h, Q\right)$ scheme}

We consider first the conservative variable $W={ }^{t}\left(Z_{f}, h, Q\right)$. Note that this solver corresponds to the initial VFRoe scheme [22]. The main interest of this interface Riemann solver is the discrete continuity of $Q$ through the stationary wave, in agreement with the Riemann invariant (13a).

If we develop the system (3), we can write the convection matrix (which identifies with the jacobian matrix of the numerical flux $F, W(W))$ :

$$
B(Y)=\left(\begin{array}{ccc}
0 & 0 & 0 \\
0 & 0 & 1 \\
c^{2} & c^{2}-u^{2} & 2 u
\end{array}\right)
$$

Eigenvalues of the matrix $B(Y)$ are :

$$
\lambda_{1}=0, \lambda_{2}=u-c, \lambda_{3}=u+c .
$$

The associated matrix of right eigenvectors is :

$$
\Omega=\left(\begin{array}{ccc}
c^{2}-u^{2} & 0 & 0 \\
-c^{2} & 1 & 1 \\
0 & u-c & u+c
\end{array}\right) .
$$

If we refer to the exact solution (19) of the linearised Riemann problem (9), we can write:

$$
W^{*}\left(0^{+} ; W_{L}, W_{R}\right)=W^{*}\left(0^{-} ; W_{L}, W_{R}\right)+\frac{\llbracket Z_{f} \rrbracket_{L}^{R}}{\tilde{c}^{2}-\tilde{u}^{2}}\left(\begin{array}{c}
\tilde{c}^{2}-\tilde{u}^{2} \\
-\tilde{c}^{2} \\
0
\end{array}\right)
$$

where $\tilde{u}=u(\widehat{Y})$ and $\tilde{c} c(\widehat{Y})$. This implies that the discharge $Q$ is continuous through the stationary wave, according to relation (13a). So, the scheme associated to $h$ is conservative. By the same way, one may write the relations to connect a state $W$ to a state $W_{a}$ through the $u-c$ wave :

$$
W=W_{a}+\frac{1}{2}\left(\frac{\tilde{c} \llbracket Z_{f} \rrbracket_{L}^{R}}{\tilde{c}-\tilde{u}}+\frac{(\tilde{c}+\tilde{u}) \llbracket h \rrbracket_{L}^{R}}{\tilde{c}}-\frac{\llbracket Q \rrbracket_{L}^{R}}{\tilde{c}}\right)\left(\begin{array}{c}
0 \\
1 \\
\tilde{u}-\tilde{c}
\end{array}\right)
$$

and the relations to connect a state $W$ to a state $W_{b}$ through the $u+c$ wave :

$$
W=W_{b}+\frac{1}{2}\left(\frac{\tilde{c} \llbracket Z_{f} \rrbracket_{L}^{R}}{\tilde{c}-\tilde{u}}+\frac{(\tilde{c}-\tilde{u}) \llbracket h \rrbracket_{L}^{R}}{\tilde{c}}+\frac{\llbracket Q \rrbracket_{L}^{R}}{\tilde{c}}\right)\left(\begin{array}{c}
0 \\
1 \\
\tilde{u}+\tilde{c}
\end{array}\right) .
$$




\subsection{The VFRoencv $\left(Z_{f}, 2 c, u\right)$ scheme}

We consider herein the change of variable $Y(W)={ }^{t}\left(Z_{f}, 2 c, u\right)$. The choice of variable $Y$ was motivated by the form of Riemann invariants associated with waves of speed $u-c$ and $u+c$ which are respectively $u+2 c$ and $u-2 c$ (see (6) and (7)). Moreover, in the flat bottom case (5), variable ${ }^{t}(2 c, u)$ provides a symmetrical convection matrix and the condition to maintain a positive intermediate sound speed is formally the same as the condition of vacuum occurence (8) (see for more details [10] and [4]).

The system (3) may be written related to $Y$ as follows :

$$
\begin{aligned}
& Z_{f, t}=0 \\
& (2 c)_{, t}+u(2 c)_{, x}+c u_{, x}=0 \\
& u_{, t}+c(2 c)_{, x}+u u_{, x}+g Z_{f, x}=0 .
\end{aligned}
$$

Note that this system is defined only if $h>0$ and focusing on smooth solutions. The convection matrix $B(Y)$ is :

$$
B(Y)=\left(\begin{array}{lll}
0 & 0 & 0 \\
0 & u & c \\
g & c & u
\end{array}\right)
$$

Eigenvalues of matrix $B(Y)$ read :

$$
\lambda_{1}=0, \lambda_{2}=u-c, \lambda_{3}=u+c .
$$

If we denote by $\Omega$ the matrix of right eigenvectors, we may write :

$$
\Omega=\left(\begin{array}{ccc}
u^{2}-c^{2} & 0 & 0 \\
g c & 1 & 1 \\
-g u & -1 & 1
\end{array}\right)
$$

The solution provided by the linearised Riemann problem verify through the stationary wave:

$$
Y^{*}\left(0^{+} ; Y_{L}, Y_{R}\right)=Y^{*}\left(0^{-} ; Y_{L}, Y_{R}\right)+\frac{\llbracket Z_{f} \rrbracket_{L}^{R}}{\tilde{u}^{2}-\tilde{c}^{2}}\left(\begin{array}{c}
\tilde{c}^{2}-\tilde{u}^{2} \\
g \tilde{c} \\
-g \tilde{u}
\end{array}\right)
$$

The relation between a state $Y$ and a state $Y_{a}$ through the $u-c$ wave may be written :

$$
Y=Y_{a}+\left(\frac{-g}{2(\tilde{u}-\tilde{c})} \llbracket Z_{f} \rrbracket_{L}^{R}+\llbracket c \rrbracket_{L}^{R}-\frac{\llbracket u \rrbracket_{L}^{R}}{2}\right)\left(\begin{array}{c}
0 \\
1 \\
-1
\end{array}\right)
$$

and the relation to connect a state $Y$ to a state $Y_{b}$ through the $u+c$ wave is :

$$
Y=Y_{a}+\left(\frac{-g}{2(\tilde{u}-\tilde{c})} \llbracket Z_{f} \rrbracket_{L}^{R}+\llbracket c \rrbracket_{L}^{R}+\frac{\llbracket u \rrbracket_{L}^{R}}{2}\right)\left(\begin{array}{l}
0 \\
1 \\
1
\end{array}\right) .
$$


One may easily note that the discharge $Q$ computed by the VFRoencv $\left(Z_{f}, 2 c, u\right)$ solver is different on both sides of the interface. Hence, the scheme (17) is not conservative according to the equation (3a). To avoid this problem, a new Finite Volume approximation of (3a) may be introduced :

$$
h_{i}^{n+1}=h_{i}^{n}-\frac{\Delta t}{2 \Delta x_{i}}\left(\left(Q_{i+1 / 2}^{-}+Q_{i+1 / 2}^{+}\right)-\left(Q_{i-1 / 2}^{-}+Q_{i-1 / 2}^{+}\right)\right)
$$

where $Q_{i+1 / 2}^{-}$and $Q_{i+1 / 2}^{+}$refer respectively to values at the left and the right side of the interface $x_{i+1 / 2}$. The scheme obtained from this approximate Riemann solver is able to deal with vacuum in the flat bottom case, according to tests provided in [4]. Moreover, some numerical results are provided in the last section with occurence of dry area on a non trivial topography.

\subsection{The VFRoencv $\left(Z_{f}, Q, \psi\right)$ scheme}

This approximate Riemann solver follows the same formalism as above. We consider herein the variable $Y(W)={ }^{t}\left(Z_{f}, Q, \psi\right)$ (with $Q=h u$ and $\psi=u^{2} / 2+g\left(h+Z_{f}\right)$ ). However, we may remark that this change of variable is not inversible, which may cause some problems to define the numerical flux. The choice of $Y$ is related to the form of the Riemann invariants associated with the null velocity wave (13).

The system (3) written related to $Y$ is :

$$
\begin{aligned}
& Z_{f, t}=0 \\
& Q_{, t}+u Q_{, x}+h \psi_{, x}=0 \\
& \psi_{, t}+g Q_{, x}+u \psi_{, x}=0 .
\end{aligned}
$$

As a result, the convection matrix $B(Y)$ is :

$$
B(Y)=\left(\begin{array}{lll}
0 & 0 & 0 \\
0 & u & h \\
0 & g & u
\end{array}\right)
$$

As above, eigenvalues of matrix $B(Y)$ are:

$$
\lambda_{1}=0, \lambda_{2}=u-c, \lambda_{3}=u+c .
$$

If $\Omega$ is the matrix of right eigenvectors, we may write:

$$
\Omega=\left(\begin{array}{ccc}
1 & 0 & 0 \\
0 & -c & c \\
0 & g & g
\end{array}\right)
$$

The approximate Riemann problem to solve is the same as (18), whose solution $Y^{*}\left(x / t ; Y_{L}, Y_{R}\right)$ is defined in (19). We have the following relation through the stationary wave:

$$
Y^{*}\left(0^{+} ; Y_{L}, Y_{R}\right)=Y^{*}\left(0^{-} ; Y_{L}, Y_{R}\right)+\left(\begin{array}{c}
\llbracket Z_{f} \rrbracket_{L}^{R} \\
0 \\
0
\end{array}\right) \text {. }
$$


Thus, the solution computed by this Riemann solver is in agreement with the Riemann invariants (13a) and (13b). Hence, this approximate Riemann solver associated with the scheme (17) is able to maintain a large class of steady states, ie those based on the Riemann invariants (13) (see remark 4). A state $Y$ may be connected to a state $Y_{a}$ through the $u-c$ wave by :

$$
Y=Y_{a}+\left(\frac{-1}{2 \tilde{c}} \llbracket Q \rrbracket_{L}^{R}+\tilde{c} \llbracket \psi \rrbracket_{L}^{R}\right)\left(\begin{array}{c}
0 \\
-\tilde{c} \\
g
\end{array}\right)
$$

and a state $Y$ is connected to a state $Y_{b}$ through the $u+c$ wave by :

$$
Y=Y_{b}+\left(\frac{1}{2 \tilde{c}} \llbracket Q \rrbracket_{L}^{R}+\tilde{c} \llbracket \psi \rrbracket_{L}^{R}\right)\left(\begin{array}{l}
0 \\
\tilde{c} \\
g
\end{array}\right)
$$

Remark 2. The convection matrix $B(Y)$ may be written in a symmetrical form, as follows :

$$
B(Y)=\left(\begin{array}{ccc}
0 & 0 & 0 \\
0 & u & h \\
0 & g & u
\end{array}\right)=\left(\begin{array}{ccc}
1 & 0 & 0 \\
0 & 1 & 0 \\
0 & 0 & h / g
\end{array}\right)^{-1}\left(\begin{array}{ccc}
0 & 0 & 0 \\
0 & u & h \\
0 & h & h u / g
\end{array}\right)
$$

Remark 3. Note that the system (10) provides a pseudo-conservative form for smooth solutions. Thus, one could use this form to define a Finite Volume scheme from it (with the VFRoencv $\left(Z_{f}, Q, \psi\right)$ solver for instance). However, one can easily verify that, even in the flat bottom case, the Rankine Hugoniot relations are not equivalent. Indeed, noting $v=u-\sigma$ ( $\sigma$ the shock speed), the jump relations provided by the (real) system in the flat bottom case (5) are:

$$
\begin{gathered}
\llbracket h v \rrbracket=0 \\
\overline{h v} \llbracket v \rrbracket+g \bar{h} \llbracket h \rrbracket=0
\end{gathered}
$$

whereas the jump relations provided by the pseudo-conservative system (10) in the flat bottom case write:

$$
\begin{gathered}
\llbracket h v \rrbracket=0 \\
\bar{v} \llbracket v \rrbracket+g \llbracket h \rrbracket=0
\end{gathered}
$$

which are not equivalent to the previous relations.

Remark 4. According to remark 1 and relations (13) (assuming that the Riemann invariants and the Rankine Hugoniot relations identify through le LD field), one can define the following discrete steady states:

$$
\begin{gathered}
\llbracket Q \rrbracket_{i}^{i+1}=0 \\
\llbracket \psi \rrbracket_{i}^{i+1}=0 .
\end{gathered}
$$

Moreover, these states strictly include steady states with $u \equiv 0$ :

$$
\begin{gathered}
u_{i}=0 \\
\llbracket h+Z_{f} \rrbracket_{i}^{i+1}=0 .
\end{gathered}
$$


Remark 5. Steady states (26) are exactly preserved by the VFRoencv $\left(Z_{f}, Q, \psi\right)$. Moreover, all VFRoencv schemes presented here preserve exactly steady states (27).

We turn now to the second class of methods based on the splitting method.

\section{Fractional step method}

We present now a new scheme, based on a fractional step method (see [24], [21] and [26]). The system (3) is split in two parts. The first one is the conservative and homogenous system of P.D.E. :

$$
\begin{aligned}
& h_{, t}+(h u)_{, x}=0 \\
& (h u)_{, t}+\left(h u^{2}+g \frac{h^{2}}{2}\right)_{, x}=0 .
\end{aligned}
$$

The second one is the system of O.D.E. :

$$
\begin{aligned}
& h_{, t}=0 \\
& (h u)_{, t}=-g h Z_{f}^{\prime}(x) .
\end{aligned}
$$

The effects of the source term are decoupled from the conservative system. So, a robust method may be applied to compute the system (28) (ensuring positivity of $h$ ), and a classical method is used to solve the O.D.E. (29).

\subsection{The VFRoencv $(2 c, u)$ scheme}

To compute the (strictly) hyperbolic, conservative and homogenous system (28), we propose the VFRoencv $(2 c, u)$ scheme (see [10] and [4]). This system may be written in terms of non conservative variable $Y(W)={ }^{t}(2 c, u)$. Hence comes :

$$
\frac{\partial Y}{\partial t}+B(Y) \frac{\partial Y}{\partial x}=0
$$

with:

$$
B(Y)=\left(\begin{array}{ll}
u & c \\
c & u
\end{array}\right)
$$

Matrix $B(Y)$ is symmetric. The intermediate state is given by (we set here $\hat{Y}=\bar{Y}$ ) :

$$
\begin{aligned}
& u_{s}=\bar{u}-\llbracket c \rrbracket_{L}^{R} \\
& c_{s}=\bar{c}-\frac{\llbracket u \rrbracket_{L}^{R}}{4}
\end{aligned}
$$


where $\llbracket \alpha \rrbracket_{L}^{R}$ represents $\alpha_{R}-\alpha_{L}$, for each interface Riemann problem. Note that the linearization has been made around the state $(2 \bar{c}, \bar{u})$.

Vacuum arises in the intermediate state of linearized Godunov solver if and only if initial data makes vacuum occur in the exact solution of the Riemann problem associated with the non linear set of equations (see the condition (8)). Actually, when focusing on the solution of the Riemann problem, vacuum may only occur when initial data is such that two rarefaction waves develop. Riemann invariants are preserved in that case, hence $u+2 c$ (respectively $u-2 c$ ) is constant in the 1-rarefaction wave (respectively the 2-rarefaction wave). Due to the specific form of the linearized system written in terms of non conservative variable $Y$, one gets from a discrete point of view :

$$
\begin{aligned}
u_{R}-2 c_{R} & =u_{s}-2 c_{s} \\
u_{L}+2 c_{L} & =u_{s}+2 c_{s} .
\end{aligned}
$$

Thus, the linearized solver is well suited to handle double rarefaction waves in the solution of the exact Riemann problem. Hence, the discrete condition to ensure the positivity of $c_{s}$ is :

$$
u_{R}-u_{L}<2\left(\sqrt{g h_{R}}+\sqrt{g h_{L}}\right)
$$

which exactly identifies with the continuous condition (8).

\subsection{The fractional step method}

The Finite Volume scheme which computes the homogenous system (28) may be written as follows:

$$
\begin{aligned}
W_{i}^{n+\frac{1}{2}}=W_{i}^{n}-\frac{\Delta t}{\Delta x_{i}} & \left(F\left(W_{i+\frac{1}{2}}^{*}\left(0 ; W_{i}, W_{i+1}\right)\right)\right. \\
- & \left.F\left(W_{i-\frac{1}{2}}^{*}\left(0 ; W_{i-1}, W_{i}\right)\right)\right)
\end{aligned}
$$

where $W_{i+\frac{1}{2}}^{*}\left(x / t ; W_{i}, W_{i+1}\right)$ is the solution of the Riemann problem at the interface $x_{i+\frac{1}{2}}$, approximated by the VFRoencv $(2 c, u)$ solver.

The system of O.D.E. (29) is approximated by an explicit Euler method for the time part, and by a centered discretisation for the space part:

$$
\begin{aligned}
h_{i}^{n+1} & =h_{i}^{n+\frac{1}{2}} \\
Q_{i}^{n+1} & =Q_{i}^{n+\frac{1}{2}}-\frac{\Delta t}{\Delta x_{i}} g h_{i}^{n+\frac{1}{2}}\left(\frac{Z_{f i+1}-Z_{f i-1}}{2}\right) .
\end{aligned}
$$

Note that the property of the VFRoencv $(2 c, u)$ scheme concerning the occurence of vacuum is not modified by step (33). Some numerical results with dry area provided in the following confirm the good behaviour of the fractional step method over vacuum.

Note that neither steady states (26) nor steady states (27) are maintained by the whole algorithm. This phenomenon is well known and will be discussed in the following, based on some numerical experiments, to emphasize that the algorithm is able to converge towards steady states. 
Remark 6. In the flat bottom case, the fractional step method (32)-(33) and the VFRoencv $\left(Z_{f}, 2 c, u\right)$ scheme presented before provide the same algorithm.

Remark 7. The two steps may be recast in one single step form, as follows :

$$
\begin{aligned}
h_{i}^{n+1} & =h_{i}^{n}-\frac{\Delta t}{\Delta x_{i}}\left(Q_{i+\frac{1}{2}}^{*}-Q_{i-\frac{1}{2}}^{*}\right) \\
Q_{i}^{n+1} & =Q_{i}^{n}-\frac{\Delta t}{\Delta x_{i}}\left(\left(h u^{2}+g h^{2} / 2\right)_{i+\frac{1}{2}}^{*}-\left(h u^{2}+g h^{2} / 2\right)_{i-\frac{1}{2}}^{*}\right)-\frac{\Delta t}{\Delta x_{i}} g h_{i}^{n+1}\left(\frac{Z_{f i+1}-Z_{f i-1}}{2}\right)
\end{aligned}
$$

where ()$_{i+\frac{1}{2}}^{*}$ denotes the variable computed by the VFRoencv $(2 c, u)$ scheme at the interface $x_{i+\frac{1}{2}}$.

\section{$5 \quad$ A higher order extension}

All schemes previously presented are derived from "first order" methods. We introduce in this section an extension to obtain more accurate results and to increase rate of convergence (related to the mesh size). This method is based on a linear reconstruction on each cell by the method introduced by B. Van Leer in [25], namely MUSCL (Monotonic Upwind Schemes for Conservation Laws). This formalism is usually applied in a conservative and homogenous framework (see [11] for numerical measures with some VFRoencv schemes on Euler system, with non smooth solutions). However, the source term of topography deeply modifies the structure of the solutions.

When applied to the shallow-water equations on a flat bottom, the MUSCL method would limit the slope of variables $h$ and $u$ for instance. However, the source term of topography must be taken into account. Indeed, refering to a steady state such that $h+Z_{f} \equiv C^{s t e}$ and $u \equiv 0$, a classical MUSCL reconstruction breaks the balance of the state. Since a general class of steady states are defined by $Q$ and $\psi$ constant (see remark 4), one may require that the reconstruction does not modify these states. Moreover, the method must be able to deal with vacuum. We present here a slope limiter which verifies these requirements.

For a sake of simplicity, all variables used in this section are supposed to be time-independant. Indeed, the MUSCL method is applied at each time step, ie $t$ is locally fixed to $t^{n}$ at the $n^{\text {th }}$ time step. Moreover, though this MUSCL method may be computed on irregular meshes, we restrict this presentation to constant space step $\Delta x$.

Some notations are first introduced. Let $\left\{\alpha_{i}\right\}_{i \in \mathbb{Z}}$ a variable, constant on each cell, where a cell is $I_{i}=\left[x_{i-1 / 2} ; x_{i+1 / 2}\right]$. Let $x_{i}=\left(x_{i+1 / 2}+x_{i-1 / 2}\right) / 2$ and $\delta_{i}(\alpha)$ the (constant) slope associated to $\alpha_{i}$ on the cell $I_{i}$. Let $\alpha_{i}^{\text {lin }}(x), x \in I_{i}$, the function defined on $I_{i}$ by :

$$
\alpha_{i}^{\text {lin }}(x)=\alpha_{i}-\delta_{i}\left(x-x_{i}\right) \quad x \in I_{i} .
$$

Thus, to compute numerical flux at an interface $x_{i+1 / 2}$, the initial data become $\alpha_{i}^{\text {lin }}\left(x_{i+1 / 2}\right)$ and $\alpha_{i+1}^{l i n}\left(x_{i+1 / 2}\right)$ of the local Riemann problem instead of $\alpha_{i}$ and $\alpha_{i+1}$. This step is the same as in the classical framework.

The modification of the algorithm to take into account the topography is thus restricted to the choice of variables for which the MUSCL reconstruction is applied to and to the computation of 
the slope $\delta_{i}$. The first variable is the momentum $Q$. A classical minmod slope limiter is used (see for instance [19]) :

$$
\delta_{i}(Q)= \begin{cases}s_{i+1 / 2}(Q) \min \left(\left|Q_{i+1}-Q_{i}\right|,\left|Q_{i}-Q_{i-1}\right|\right) / \Delta x & \text { if } s_{i-1 / 2}(Q)=s_{i+1 / 2}(Q), \\ 0 & \text { else, }\end{cases}
$$

where

$$
s_{i+1 / 2}(\alpha)=\operatorname{sign}\left(\alpha_{i+1}-\alpha_{i}\right) .
$$

Such a slope limiter is TVD (Total Variation Diminishing) in the following sense :

Property 1. Let $\Omega$ an open subset of $\mathbb{R}$ (here $\Omega=\mathbb{R}$ ).

Let us define the total variation of a function $v \in L_{l o c}^{1}(\Omega)$ :

$$
\|v\|=\sup \left\{\int_{\Omega} v \operatorname{div} \phi d x, \phi \in \mathcal{C}_{0}^{1}(\Omega),\|\phi\|_{L^{\infty}(\Omega)} \leq 1\right\} .
$$

If $v^{c s t}$ and $v^{\text {lin }}$ are the functions which respectively represent the constant and linear piecewise approximations of $v$ :

$$
\begin{aligned}
& v^{c s t}(x)=v_{i} \quad i \in \mathbb{Z} \text { such that } x \in I_{i}, \\
& v^{\text {lin }}(x)=v_{i}^{\text {lin }} \quad i \in \mathbb{Z} \text { such that } x \in I_{i},
\end{aligned}
$$

then $v^{\text {lin }}$ defined by the minmod slope limiter verifies:

$$
\left\|v^{\text {lin }}\right\| \leq\left\|v^{c s t}\right\| .
$$

The linear reconstruction on $Q$ based on (34) verifies property 1 .

As mentionned above, stationary states must be preserved by the method, in order to permit convergence in time to steady states. To satisfy this requirement, one may choose to apply the reconstruction on $\psi$ and to verify the property 1 for $\psi$. However, the change of variable from $(h, Q)$ to $(Q, \psi)$ is not inversible. Thus, the slope limitation is made on the water height, but the computation of the slope $\delta_{i}(h)$ is modified to take into account $\psi$. Let us first define :

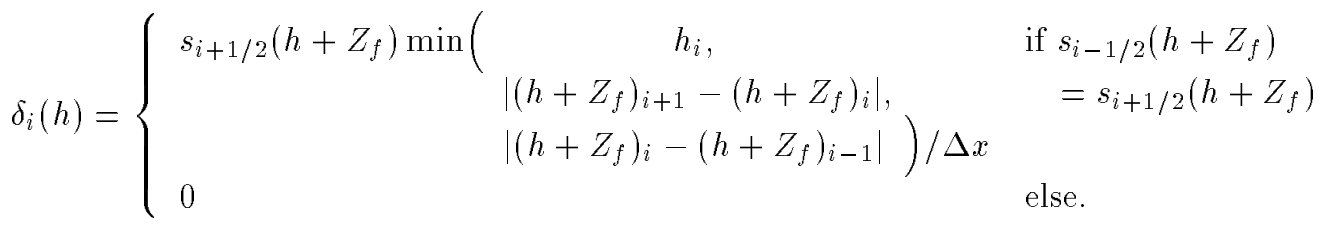

The term $h_{i}$ in the minimum enables the method to deal with vacuum. The profile of $\psi$ does not appear in the computation of $\delta_{i}(h)$ (though $\psi$ and $g\left(h+Z_{f}\right)$ identify when $u \equiv 0$ ). Hence, when the source term is locally non null (ie $Z_{f i-1} \neq Z_{f i}$ or $Z_{f i} \neq Z_{f i+1}$ ), $\delta_{i}(h)$ must be modified, according to values of $\psi_{i-1}, \psi_{i}$ and $\psi_{i+1}$. Since the slope limiters are based on a TVD requirement for the linear reconstruction, we impose a TVD-like condition on $\psi$, for the computation of $\delta_{i}(h)$. Let $\Psi$ be the function :

$$
\Psi\left(Z_{f}, h, Q\right)=\frac{Q^{2}}{2 h^{2}}+g\left(h+Z_{f}\right)
$$


All methods presented in this paper use the following values, $\forall i \in \mathbb{Z}$ :

$$
\begin{aligned}
\Psi_{i}^{-} & =\Psi\left(Z_{f i}, h_{i}-\delta_{i}(h) \frac{\Delta x}{2}, Q_{i}-\delta_{i}(Q) \frac{\Delta x}{2}\right), \\
\Psi_{i} & =\Psi\left(Z_{f i}, h_{i}, Q_{i}\right) \quad\left(=\psi_{i}\right), \\
\Psi_{i}^{+} & =\Psi\left(Z_{f i}, h_{i}+\delta_{i}(h) \frac{\Delta x}{2}, Q_{i}+\delta_{i}(Q) \frac{\Delta x}{2}\right) .
\end{aligned}
$$

Following these notations, $\Psi_{i}$ is the value of $\Psi$ at the center of each cell $I_{i}, \Psi_{i}^{-}$is the value of $\Psi$ at the right of each interface $x_{i-1 / 2}$ and $\Psi_{i}^{+}$is the value of $\Psi$ at the left of each interface $x_{i+1 / 2}$, $i \in \mathbb{Z}$. The computation of numerical flux at an interface $x_{i+1 / 2}$ needs $\Psi_{i}^{+}$and $\Psi_{i+1}^{-}$. Following notations previously introduced, let $Z_{f}^{\text {cst }}, h^{\text {cst }}$ and $Q^{\text {cst }}$ be the piecewise constant approximations and let $Z_{f}^{l i n}, h^{l i n}$ and $Q^{l i n}$ be the piecewise linear approximations. Thus, one can easily verify that $\left\|\Psi\left(Z_{f}^{\text {lin }}, h^{\text {lin }}, Q^{\text {lin }}\right)\right\|$ is not less or equal to $\left\|\Psi\left(Z_{f}^{c s t}, h^{c s t}, Q^{c s t}\right)\right\|$. Hence, the reconstructions (34) and (36) do not imply that $\Psi$ verifies property 1 . An idea to solve this problem should be limiting "strongly" $h$ (ie computing $\left.\delta_{i}(h)=0\right)$ if $\Psi\left(Z_{f}^{\text {lin }}, h^{\text {lin }}, Q^{\text {lin }}\right)$ does not verify the TVD requirement. However, this condition may be considered too restrictive. Thus, we introduce the following condition :

$$
\begin{aligned}
& 0 \leq\left|\Psi_{i}-\Psi_{i}^{-}\right| \leq\left|\Psi_{i}-\Psi_{i-1}\right| / 2, \\
& 0 \leq\left|\Psi_{i}^{+}-\Psi_{i}\right| \leq\left|\Psi_{i+1}-\Psi_{i}\right| / 2,
\end{aligned}
$$

illustrated by figure 2 .

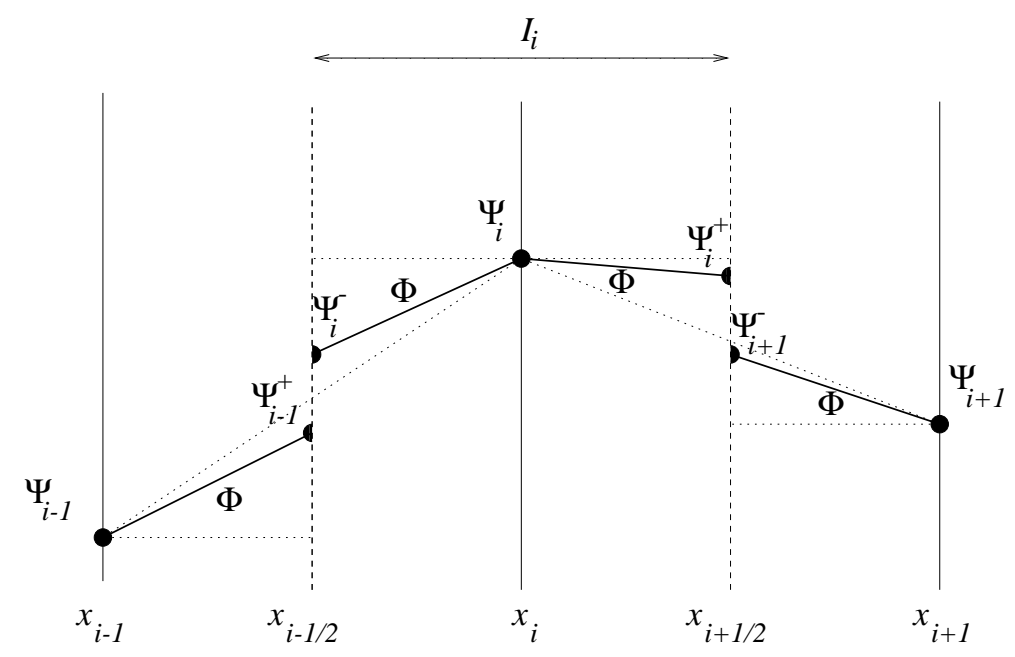

Figure 2: a TVD-like reconstruction for $\Psi$

Condition (37) imposes a TVD-like condition on $\Psi$. Indeed, assuming that $\Psi_{i}^{-}, \Psi_{i}$ and $\Psi_{i}^{+}$fulfill condition (37) $\forall i \in \mathbb{Z}$, if $\Phi$ denotes the linear interpolation computed from $\Psi_{i}^{-}, \Psi_{i}$ and $\Psi_{i}^{+} \forall i \in \mathbb{Z}$, then $\Phi$ verifies the following TVD property :

$$
\|\Phi\| \leq\left\|\Psi\left(Z_{f}^{c s t}, h^{c s t}, Q^{c s t}\right)\right\|,
$$


which may be seen as the counterpart of (35).

We recall now all the steps of the algorithm used to compute slopes $\delta_{i}(h)$ and $\delta_{i}(Q), \forall i \in \mathbb{Z}$ :

1. Computation of $\delta_{i}(Q)$ :

$$
\delta_{i}(Q)= \begin{cases}s_{i+1 / 2}(Q) \min \left(\left|Q_{i+1}-Q_{i}\right|,\left|Q_{i}-Q_{i-1}\right|\right) / \Delta x & \text { if } s_{i-1 / 2}(Q)=s_{i+1 / 2}(Q) \\ 0 & \text { else }\end{cases}
$$

2. Computation of $\delta_{i}(h)$ :

- if $Z_{f i-1}=Z_{f i}=Z_{f i+1}$, then the minmod slope limiter is applied to compute $\delta_{i}(h)$ :

$$
\delta_{i}(h)= \begin{cases}s_{i+1 / 2}(h) \min \left(\left|h_{i+1}-h_{i}\right|,\left|h_{i}-h_{i-1}\right|\right) / \Delta x & \text { if } s_{i-1 / 2}(h)=s_{i+1 / 2}(h), \\ 0 & \text { else, }\end{cases}
$$

- else, $\delta_{i}(h)$ is first computed by a classical minmod limiter on $h+Z_{f}$ :

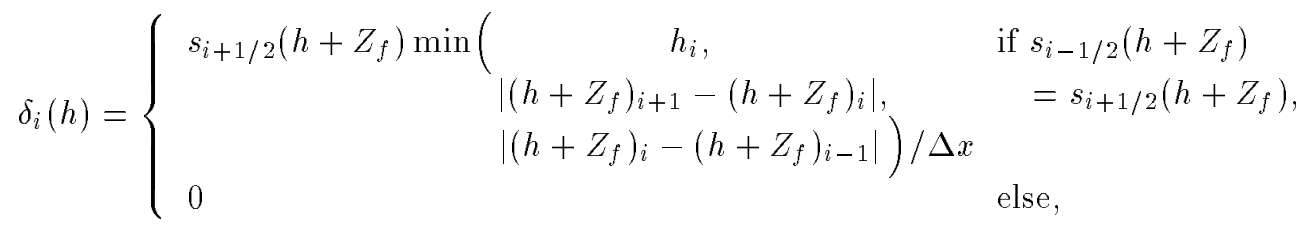

- but if condition (37) is not fulfilled, then we reset $\delta_{i}(h)$ to

$$
\delta_{i}(h)=0 .
$$

Let us emphasize that, when $\delta_{i}(h)$ is set to 0 , conditions (37) may not be verified (because of the limitation on $Q$ ).

Some numerical results are described in the following and point out the good behaviour of the slope limiter obtained. This slope limiter is combined with a second order Runge-Kutta integration wrt time.

\section{Numerical results}

Though several VFRoencv schemes have been previously discussed, only numerical results performed by the VFRoencv $\left(Z_{f}, 2 c, u\right)$ scheme with the higher order extension and by the fractional step method are presented here (some complementary tests are provided in appendix). Some experiments tested herein come from a workshop on dam-break wave simulation [15]. Most of them deal with steady states on non trivial bottom. The ability of the methods to compute dry area is tested too. Let us emphasize that all the numerical results have been obtained without any "clipping" treatment (ie non-physical values like negative water height are not artificially set to 0 ). 
The first four tests are performed with the same topography. The channel length is $l=25 m$. The bottom $Z_{f}$ is defined as follows :

$$
Z_{f}(x)= \begin{cases}1,2-1,15(x-10)^{2} & \text { if } 8 m<x<12 m \\ 0 & \text { else }\end{cases}
$$

Only initial and boundary conditions are modified.

All tests cases are computed with a $C F L$ number set to 0,4 .

\subsection{Flow at rest}

The initial condition of this test case is a flow at rest. Thus, numerically, it fulfills conditions (27), where $h>0$. Since we compute a flow at rest, we impose $h+Z_{f}=\max \left(Z_{f} ; 0,15\right) m$ and $Q=0 \mathrm{~m}^{2} / \mathrm{s}$ all along the mesh, which is composed by 300 nodes. As expected, the VFRoencv

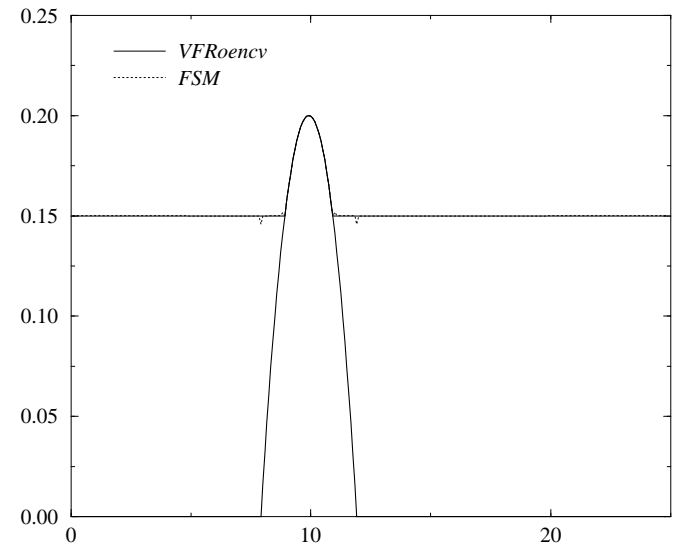

Figure 3: Flow at rest : water height

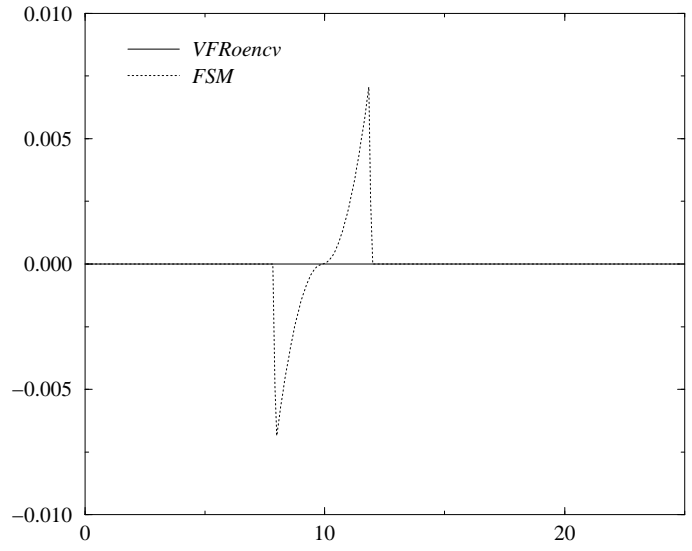

Figure 4: Flow at rest : discharge

scheme exactly preserves the steady state (figures 3 and 4). Moreover, though it is not plotted here, we may emphasize that the behaviour of this scheme stays as good as in this case when the initial conditions are $h+Z_{f}=0,5 m$ (no dry cells) or $h=0 m$ (no water). The fractional step method (FSM) does not maintain $h+Z_{f}$ and $Q$ constant on the wet cells. The slope of topography introduces a convection of water. The fractional step method nonetheless converges towards the right solution when the mesh is refined.

The interest of the next three tests (extracted from [15]) is to study the convergence of this scheme towards a steady state. All these tests are performed on 300 cells. The boundary conditions are a positive imposed discharge $Q_{\text {in }}$ on the left bound, and a imposed height $h_{\text {out }}$ on the right bound (except in the case of a supercritical flow). The initial condition is set to $h=h_{\text {out }}$ and $Q=0$. All results are plotted at $T_{M A X}=200 s$ (and $C F L=0,4$ ). To discuss results, several profiles are plotted, namely $h, Q$, and $\psi$. Moreover, to illustrate the quantitative convergence of the methods, 
the normalised time variation in $L^{2}$-norm is plotted too (see figure 6 for instance) : time $t$ in seconds for $x$-axis and $\ln \frac{\left\|h^{n+1}-h^{n}\right\|_{L^{2}}}{\left\|h^{3}-h^{2}\right\|_{L^{2}}}$ for $y$-axis.

\subsection{Subcritical flow over a bump}

Here, the boundary conditions are $h_{\text {out }}=2 \mathrm{~m}$ and $Q_{\text {in }}=4,42 \mathrm{~m}^{2} / \mathrm{s}$. The two solutions provided by the VFRoencv scheme and the fractional step method seem very close to each other, according to figure 5 (they are in agreement with the analytic solution). However, figures 7 and 8 focus on

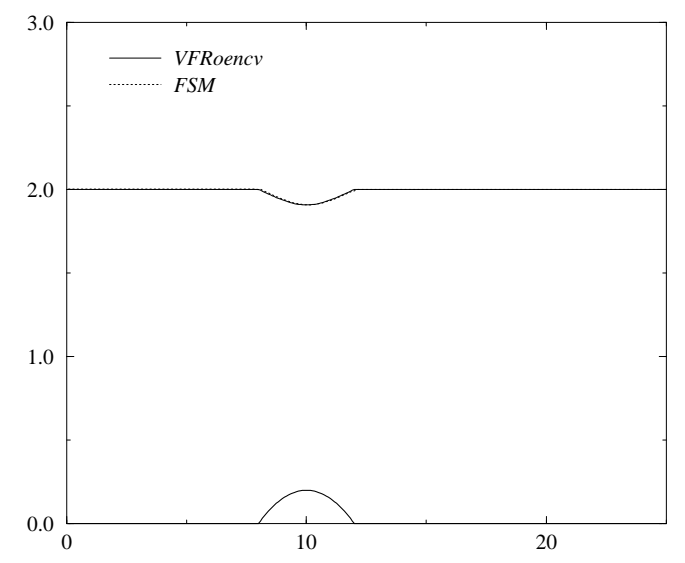

Figure 5: Subcritical flow : water height

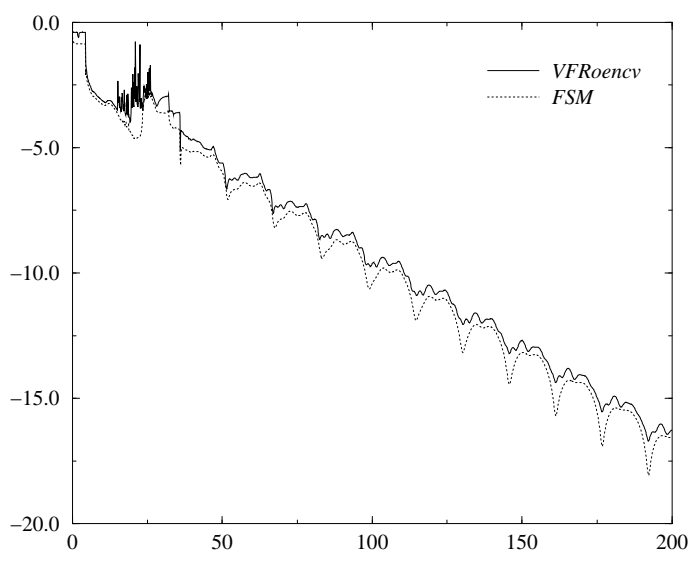

Figure 6: Subcritical flow : normalised time variation in $L^{2}$-norm

some differences between the two methods: whereas $Q$ and $\psi$ seem to be constant in the case of the VFRoencv scheme, the fractional step method makes occur oscillations near variations of topography. The two profiles on figure 6 are superposed, and show that the two methods converge to steady state.

\subsection{Transcritical flow over a bump}

The boundary conditions are $Q_{\text {in }}=1,53 \mathrm{~m}^{2} / \mathrm{s}$ and $h_{\text {out }}=0,66 \mathrm{~m}$. The analytic solution of this test is smooth, with a decreasing part, beginning at the top of the bump, with a critical (sonic) point on the decreasing part of $h$. Figure 9 shows that results provided by the VFRoencv scheme and the fractional step method are similar and the critical point implies no problem (though methods are based on approximate Godunov schemes). According to figure 10, the time variation of the VFRoencv scheme decreases more slowly than the one of the FSM. On figures 11 and 12 , one may remark that results performed by the VFRoencv scheme are more accurate, since $Q$ and $\psi$ seem almost constant. 


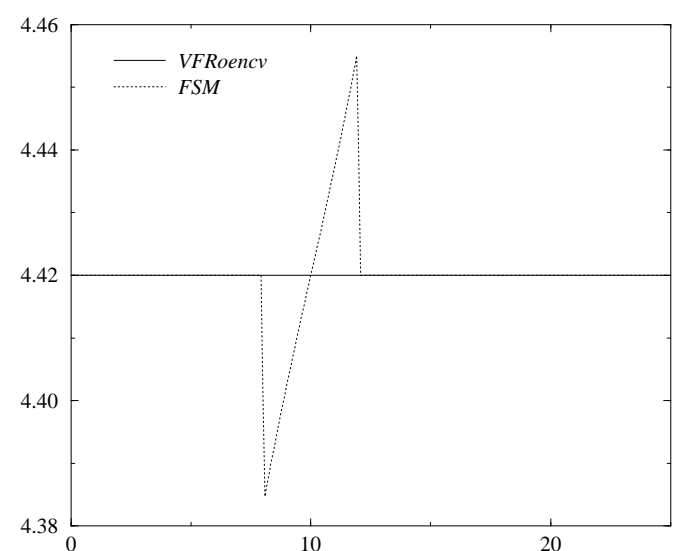

Figure 7: Subcritical flow : discharge

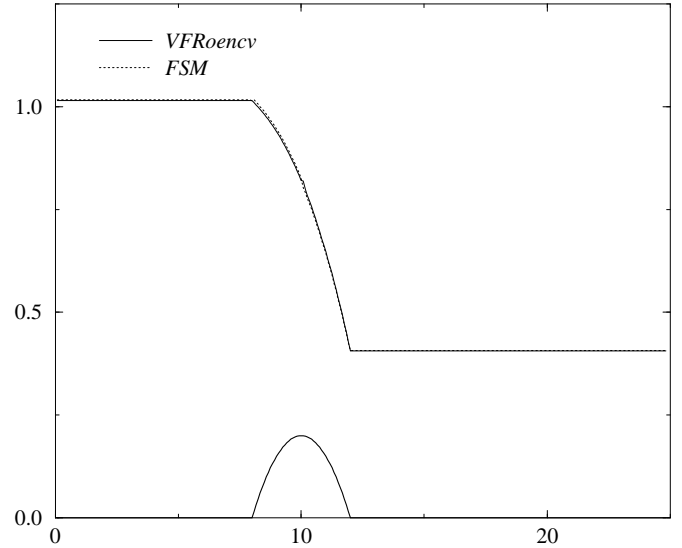

Figure 9: Transcritical flow : water height

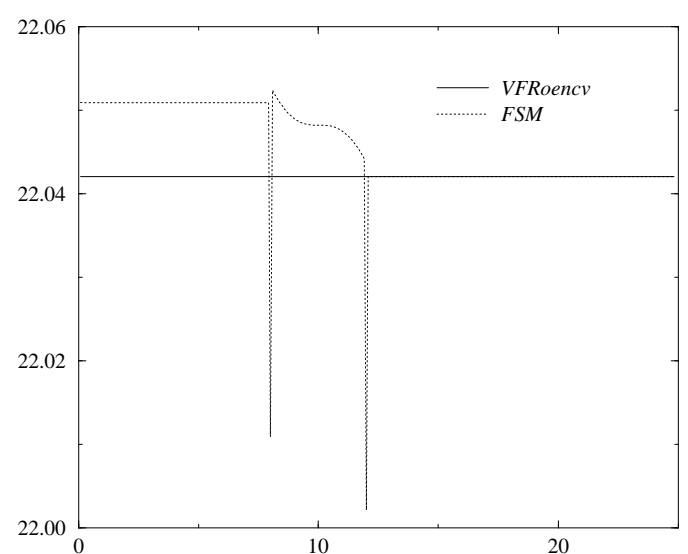

Figure 8: Subcritical flow : $\psi$

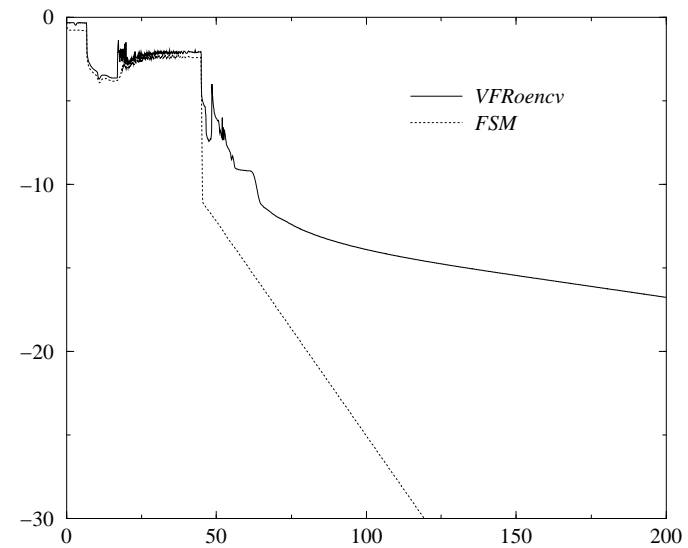

Figure 10: Transcritical flow : normalised time variation in $L^{2}$-norm 


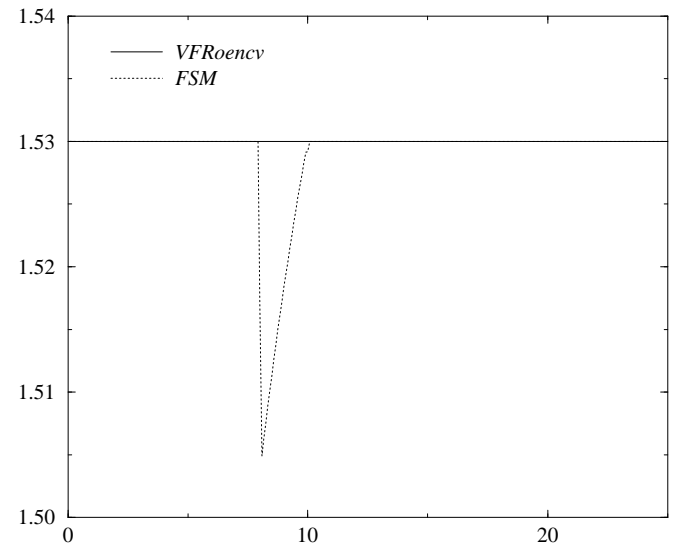

Figure 11: Transcritical flow : discharge

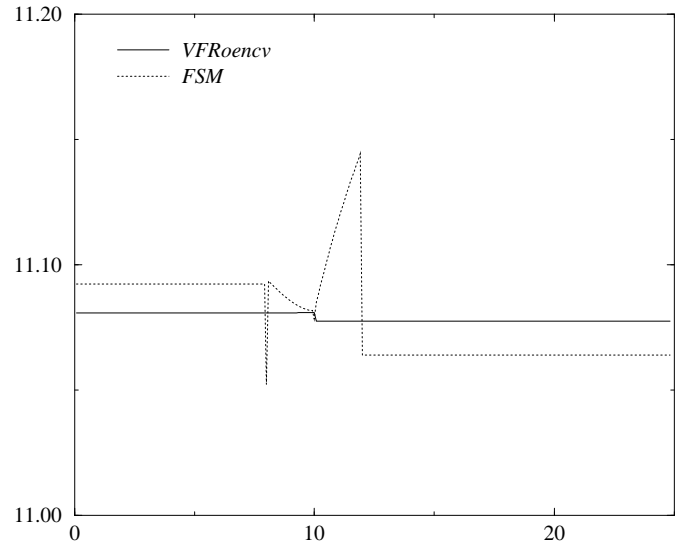

Figure 12: Transcritical flow : $\psi$

\subsection{Drain on a non flat bottom}

The topography of this test case is the same as all cases previously presented. The left boundary condition is a "mirror state"-type condition, and the right boundary condition is an outlet condition on a dry bed [5]. The initial condition is set to $h+Z_{f}=0,5 \mathrm{~m}$ and $Q=0 \mathrm{~m}^{2} / \mathrm{s}$. The solution of

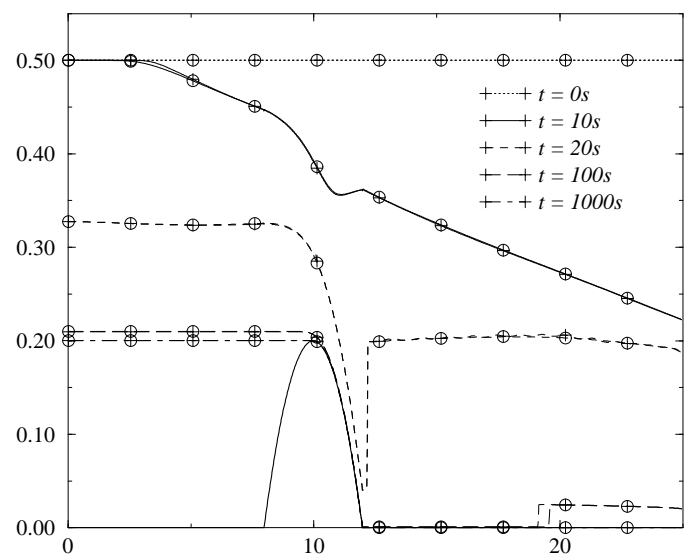

Figure 13: Drain on a non flat bottom : water height

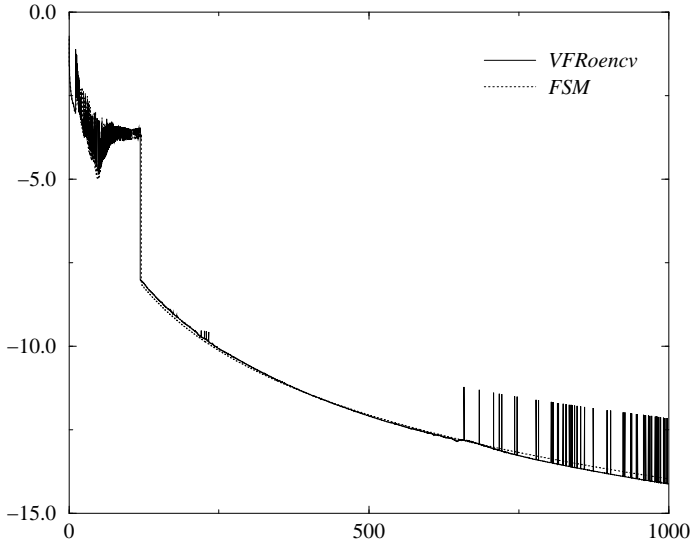

Figure 14: Drain on a non flat bottom : normalised time variation in $L^{2}$-norm

this test case at $t=+\infty$ is a state at rest on the left part of top of the bump with $h+Z_{f}=0,2 \mathrm{~m}$ and $Q=0 \mathrm{~m}^{2} / \mathrm{s}$ and a dry state (ie $h=0 \mathrm{~m}$ and $Q=0 \mathrm{~m}^{2} / \mathrm{s}$ ) on the right side of the bump. Results are presented at several times : $t=0,10,20,100$ and $1000 \mathrm{~s}$ on figures 13,15 and 16 . Note that, since a dry zone is expected at the downstream side of the bump, variable $\psi$ is not defined in this zone (thus, results plotted on figure 16 in this zone must not be taken in account). Figure 13 represents the water height computed by the VFRoencv scheme ("plus") and the fractional 


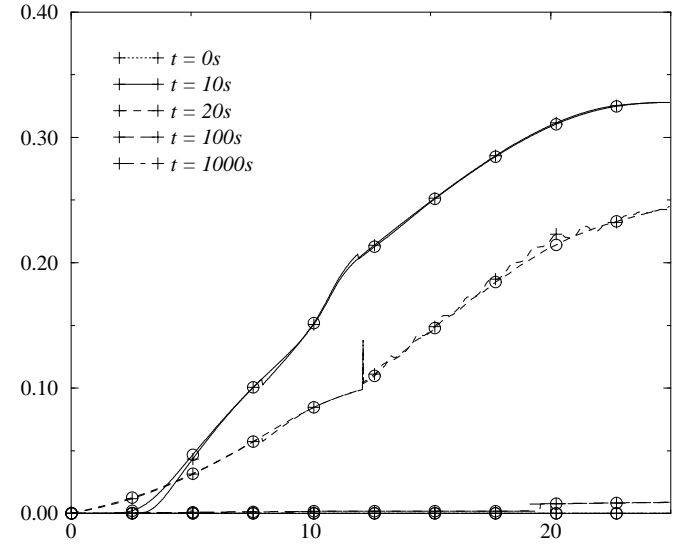

Figure 15: Drain on a non flat bottom : discharge

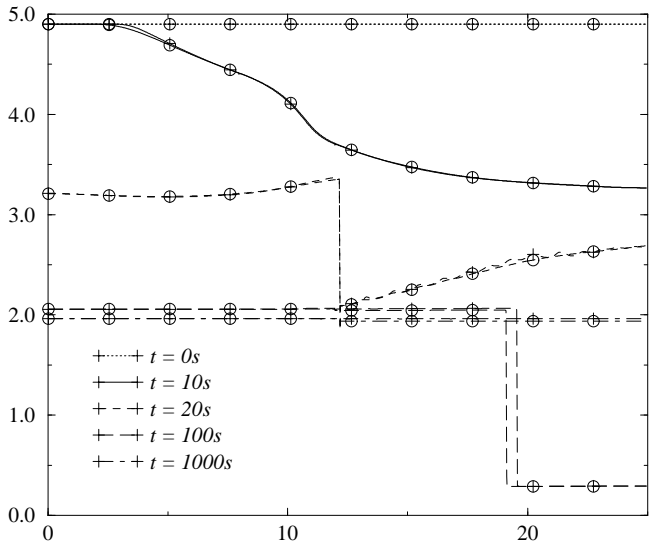

Figure 16: Drain on a non flat bottom : $\psi$

step method ("circle"). Results at intermediate times are slightly different, but denote the same behaviour. However, if the final time $T_{M A X}$ is increased, the fractional step method computes, at the left of the bump, a level of water slightly lower than the level expected, namely $h+Z_{f}=0,2 \mathrm{~m}$. This numerical phenomenon has already been pointed out by A.Y. LeRoux [18]. It is due to the non preservation of discrete steady states (27) by the fractional step method. Note however that, when the mesh is refined, the level computed tends to $h+Z_{f}=0,2 \mathrm{~m}$. Results performed by the VFRoencv scheme are good, the expected steady state is well approximated, as shown on figures 13, 15 and 16. Furthermore, the time variation is decreasing for both methods.

\subsection{Vacuum occurence by a double rarefaction wave over a step}

This numerical test is different from previous tests. Indeed, we do not study here the convergence towards a steady state but the ability of the numerical scheme to compute vacuum (ie dry bed). Moreover, the topography is not smooth (which indeed is not in agreement with initial assumptions). This test is based on a test proposed by E.F. Toro [15], but we introduce here a non trivial topography : $Z_{f}=1 \mathrm{~m}$ if $25 / 3 m<x<12,5 \mathrm{~m}$, and $Z_{f}=0 \mathrm{~m}$ otherwise (the total length is still $25 \mathrm{~m}$ ). The initial water height is initialised to $10 \mathrm{~m}$ and the initial discharge is set to $-350 \mathrm{~m}^{2} / \mathrm{s}$ if $x<50 / 3 \mathrm{~m}$ and to $350 \mathrm{~m}^{2} / \mathrm{s}$ otherwise. Several times are presented : $0 \mathrm{~s}, 0,05 \mathrm{~s}, 0,25 \mathrm{~s}, 0,45 \mathrm{~s}$ and $0,65 \mathrm{~s}$. In the case of a flat bottom, the solution would be composed by two rarefaction waves, with a dry zone occuring between the two waves. Here, since the the topography is not flat, the two algorithms introduce waves, located on the jumps of topography (see figures 17 and 18, where sign "plus" represents the VFRoencv scheme and the sign "circle" represents the fractional step method). Moreover, one may note that results computed by the two methods are close to each other, but more diffusive for the FSM method (since no MUSCL reconstruction has been performed for this algorithm). 


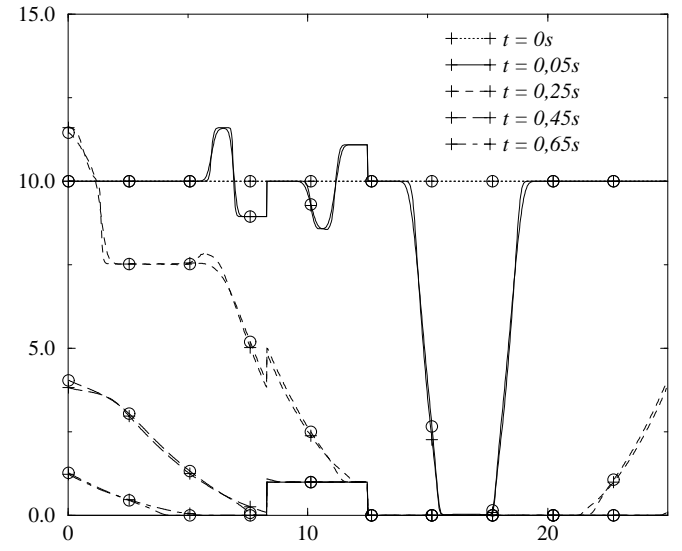

Figure 17: Vacuum occurence over a step : water height

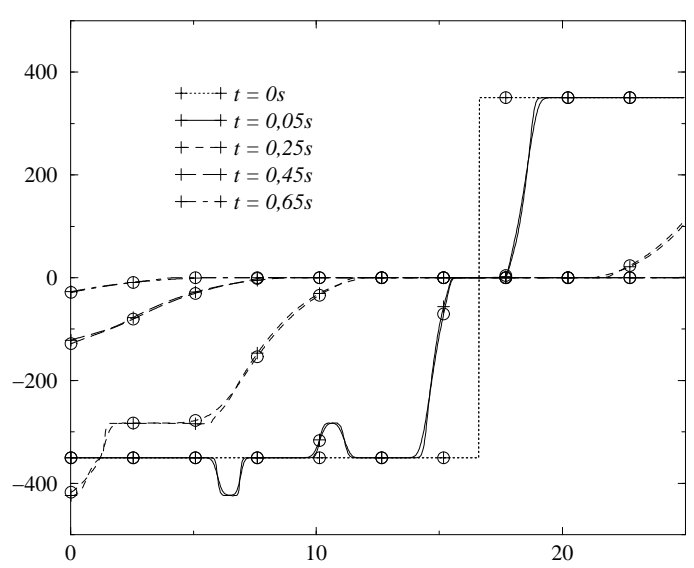

Figure 18: Vacuum occurence over a step : discharge

\section{Conclusion}

Some Finite Volume schemes have been studied in this paper to compute shallow-water equations with topography. Some relations of the system have been recalled, in the case of a piecewise constant function to approximate the topography. So, according to this approximation, several Finite Volume schemes have been introduced, based on the VFRoencv formalism [5], [11], namely the VFRoencv schemes, in variable $\left(Z_{f}, h, Q\right),\left(Z_{f}, 2 c, u\right)$ and $\left(Z_{f}, Q, \psi\right)$. All the previous schemes are able to maintain steady states with $u \equiv 0$ and the latter one can preserve a larger class of steady states. Moreover, a fractional step method based on the VFRoencv $(2 c, u)$ scheme (initially proposed in [4]) is presented. A higher order extension is also exposed, based on the minmod slope limiter, which takes into account steady states.

Numerical tests are performed with the VFRoencv $\left(Z_{f}, 2 c, u\right)$ scheme (with the higher order extension in space and a second order Runge-Kutta time integration) and the "first order" fractional step method (other tests are provided in appendix). Most of the test cases, extracted from [15], simulate the convergence in time to a steady states. The numerical schemes provide as accurate results as most of schemes tested in [15], without any significant difference between the two schemes (except for some steady states which are not strictly preserved by the fractional step method). Moreover, occurence of vacuum (dry area) on non trivial topography is also tested. The good behaviour of the two methods may be noted too in these cases, though no "clipping" treatment has been performed (ie no non-conservative treatment of negative water heights has been computed).

Considering results performed by the Well-Balanced scheme, the accuracy expected is shown on some tests. This scheme has been compared with the VFRoencv $\left(Z_{f}, 2 c, u\right)$ scheme and numerical results confirm the good behaviour of the latter scheme. However, the Well-Balanced scheme is (several times) more expensive than a usual Godunov method, since the resolution of the Riemann problem is not obvious and many configurations must be considered (this essential difficulty is due to the stationary wave). Indeed, the CPU time required by the higher order VFRoencv $\left(Z_{f}, 2 c, u\right)$ 
scheme is between 10 and 100 times lower than the CPU time required by the "first" order WellBalanced scheme.

We have presented too the VFRoe scheme (in variable $\left(Z_{f}, h, Q\right)$ ), with some results provided in appendix. The behaviour of this scheme is as good as the VFRoencv $\left(Z_{f}, 2 c, u\right)$ scheme. However, contrary to the VFRoencv $\left(Z_{f}, 2 c, u\right)$ scheme, this method fails to deal with occurence of a critical point, provided by an upstream boundary condition. Such a drawback has been emphasized too with the R.J. LeVeque scheme [20].

An interesting extension of the method presented here is to take into account a variable section $S(x, h)$ in the one-dimensional framework. The same technique may be used to approximate the corresponding source term.

\section{Acknowlegments}

This work was supported by EDF (Électricité de France) under grant C02770/AEE2704. Computational facilities were provided by EDF.

\section{A Comparison with the Well-Balanced scheme}

This appendix is devoted to the numerical comparison of the VFRoencv $Z_{f}, 2 c, u$ ) scheme with the Well-Balanced scheme presented in [18]. Note that the VFRoencv scheme is computed with the higher order extension and a second order Runge-Kutta method whereas the Well-Balanced scheme tested is the original "first" order scheme. Two tests are presented : a subcritical flow over a bump and a trancritical flow over a bump. The same topography is used for both tests :

$$
Z_{f}(x)= \begin{cases}1,2-1,15(x-10)^{2} & \text { if } 8 m<x<12 m \\ 0 & \text { else }\end{cases}
$$

Moreover, all results are plotted at $T_{M A X}=200 \mathrm{~s}$. The $C F L$ number is set to 0,4 . Computations are performed on a mesh with 300 nodes. Only initial and boundary conditions differ between the two following tests.

\section{A.1 Subcritical flow over a bump}

This test computes a transient flow, which tends to become a steady subcritical flow (see test 6.2). The imposed boundary conditions are $Q_{i n}=4,42 \mathrm{~m}^{2} / \mathrm{s}$ and $h_{\text {out }}=2 \mathrm{~m}$. The initial conditions are $Q(t=0, x)=0 \mathrm{~m}^{2} / \mathrm{s}$ and $h(t=0, x)=h_{\text {out }} m$. Figure 19 represents the water height. Results performed by the two schemes are very close to each other. The normalised variation is plotted on figure 20. The $x$-axis is the time and the $y$-axis is $\ln \frac{\| h^{n+1}-h^{n}||_{L^{2}}}{\left\|h^{3}-h^{2}\right\|_{L^{2}}}$. One can remark that the two profiles are similar and both methods provide a stationary result. This confirms the good behaviour of the VFRoencv scheme. Figures 21 and 22 present $Q$ and $\psi$. Whereas figure 21 shows that the two methods provide almost the same result, one can denote that the two profiles are slightly different. The analytic solution is $\psi=22,04205$. The slightly different value provided by 


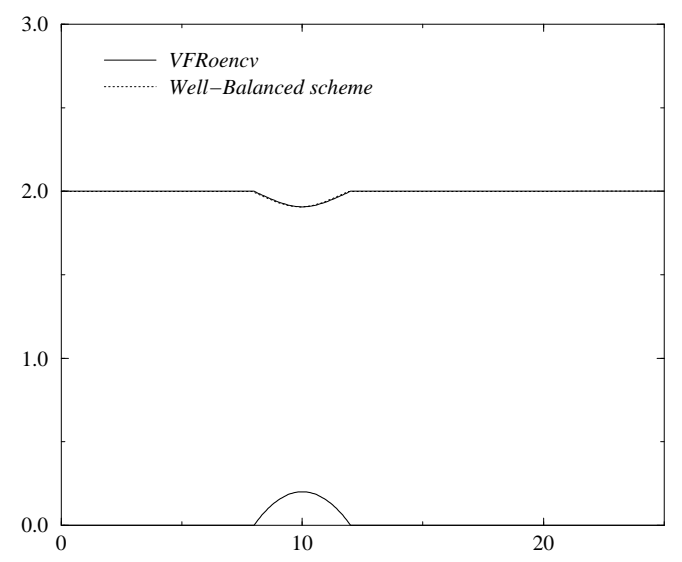

Figure 19: Subcritical flow : water height

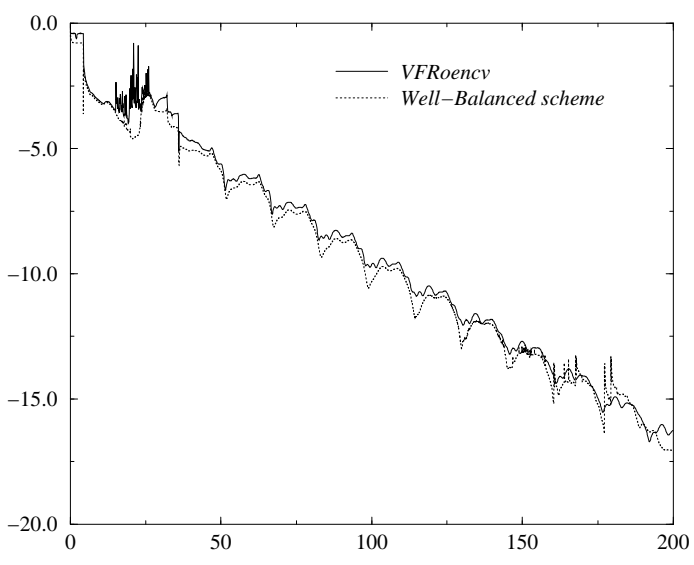

Figure 20: Subcritical flow : normalised time variation in $L^{2}$-norm

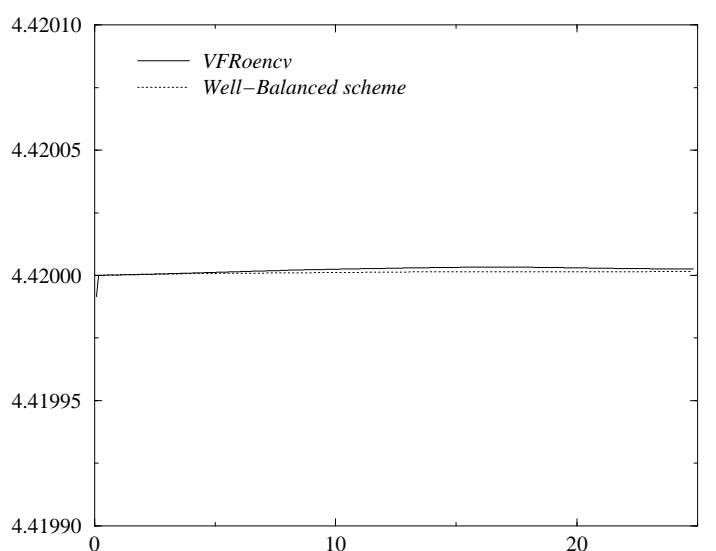

Figure 21: Subcritical flow : discharge

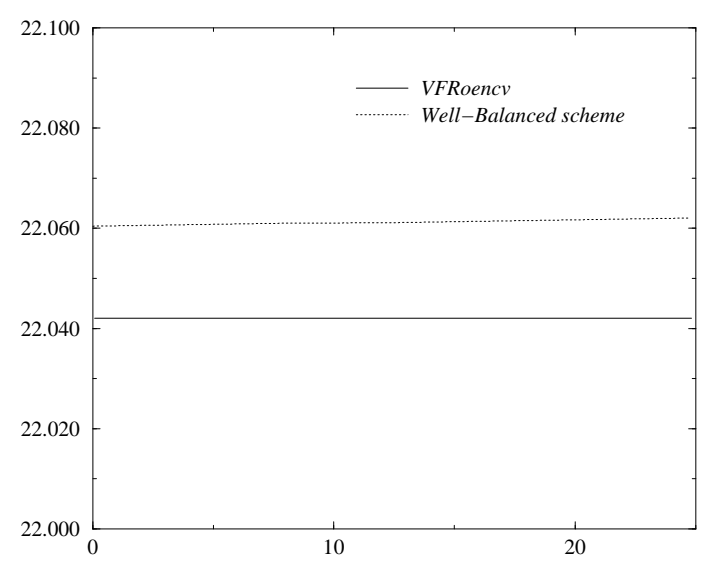

Figure 22: Subcritical flow : $\psi$ 
the Well-Balanced scheme is due to iterative methods (Newton, dichotomy, ...) used to compute the exact solution of each interface Riemann problem. Indeed, these methods stop when the relative error is $10^{-5}$ or when the number of iterations is larger than 500 .

\section{A.2 Transcritical flow over a bump}

The solution of this test case is a regular profile for the water height, with a subcritical flow upstream of the bump and a supercritical flow downstream of the bump (see test 6.3). The

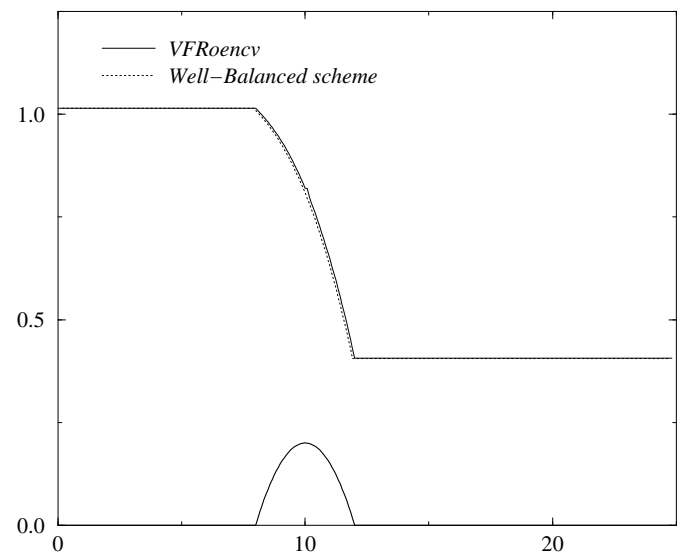

Figure 23: Transcritical flow : water height

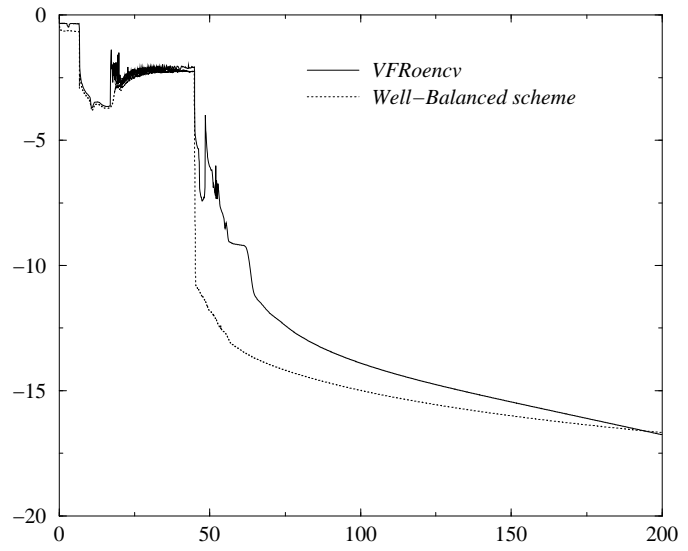

Figure 24: Transcritical flow : normalised time variation in $L^{2}$-norm

boundary conditions are $Q_{\text {in }}=1,53 \mathrm{~m}^{2} / \mathrm{s}$ and $h_{\text {out }}=0,66 \mathrm{~m}$. The initial conditions are $Q(t=$ $0, x)=0 \mathrm{~m}^{2} / \mathrm{s}$ and $h(t=0, x)=h_{\text {out }} \mathrm{m}$. Both profiles plotted on figure 23 provide a good

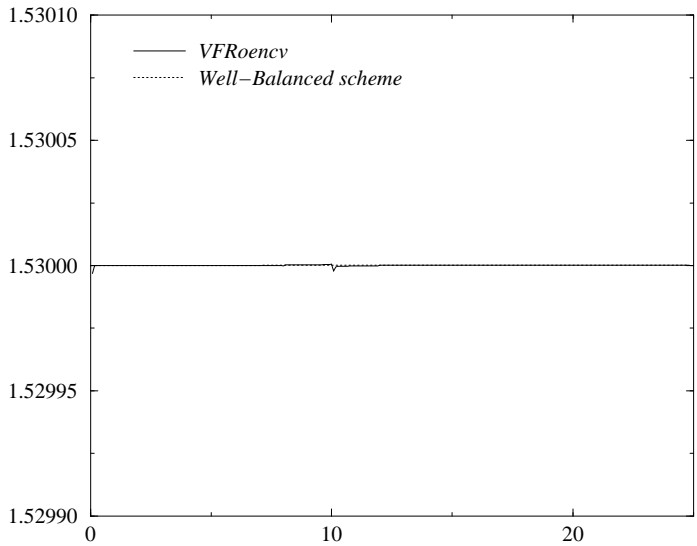

Figure 25: Transcritical flow : discharge

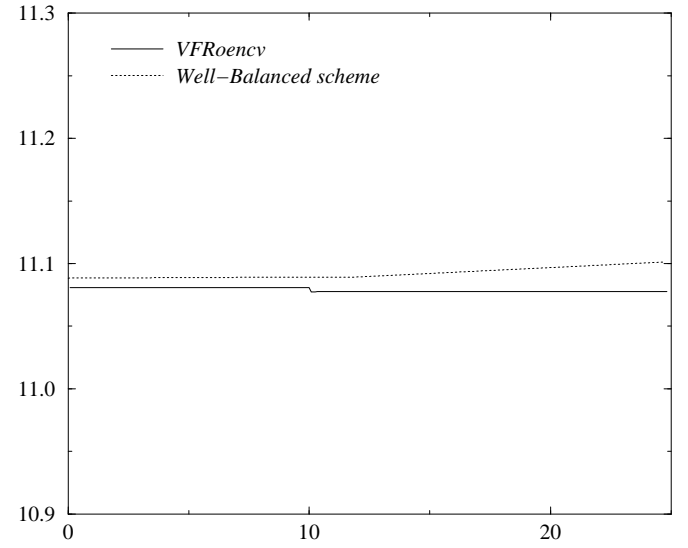

Figure 26: Transcritical flow : $\psi$ 
approximation of the expected steady solution. Moreover, figure 24 shows that the two schemes compute almost stationary solutions at $t=T_{M A X}$. Figure 25 shows that variable $Q$ is accurately computed by both methods. Moreover, figure 26, which represents variable $\psi$, denotes a slight difference between the two methods, as it has already been noticed in the previous test case.

This appendix confirms the good behaviour of the VFRoencv $\left(Z_{f}, 2 c, u\right)$ scheme. Indeed, results provided by this method with the higher order extension are very close to those provided by the Well-Balanced scheme for the two presented test cases. Moreover, the CPU time required by the VFRoencv scheme (with a second order Runge-Kutta time integration and the higher order extension) is between 10 and 100 times lower than the CPU time required by the "first" order WellBalanced scheme (no accurate CPU measurement might be done, because different computers and different languages have been used to program the methods; no optimization has been searched for the Well-Balanced scheme; the accuracy and the CPU time of the Well-Balanced scheme deeply depends on the achieves convergence of iterative methods in the exact interface Riemann solver).

\section{B Comparison with the VFRoe $\left(Z_{f}, h, Q\right)$ scheme}

We present here a numerical test performed with the VFRoe $(Z f, h, Q)$ scheme, with the higher order extension previously presented and a second order Runge-Kutta time approximation. The test case performed is the subcritical flow over a bump (see test 6.2). Let us recall the configuration of this test. The topography is :

$$
Z_{f}(x)= \begin{cases}1,2-1,15(x-10)^{2} & \text { if } 8 m<x<12 m \\ 0 & \text { else }\end{cases}
$$

The boundary conditions are $Q_{\text {in }}=4,42 \mathrm{~m}^{2} / \mathrm{s}$ and $h_{\text {out }}=2 \mathrm{~m}$. The initial conditions are

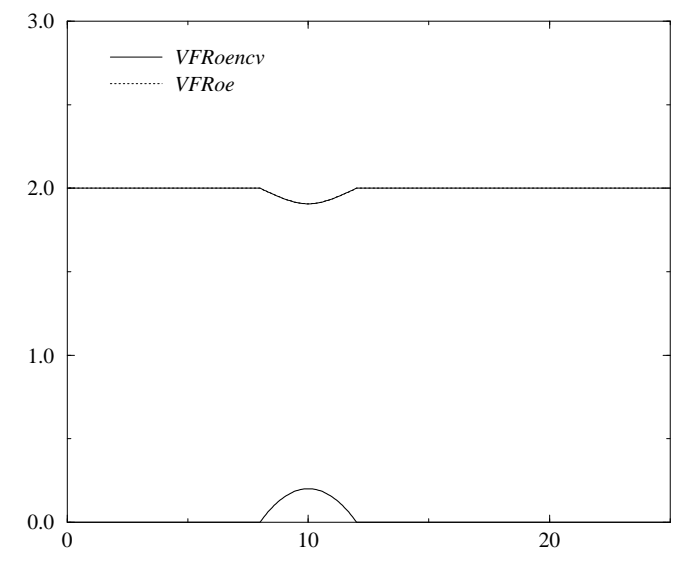

Figure 27: Subcritical flow : water height

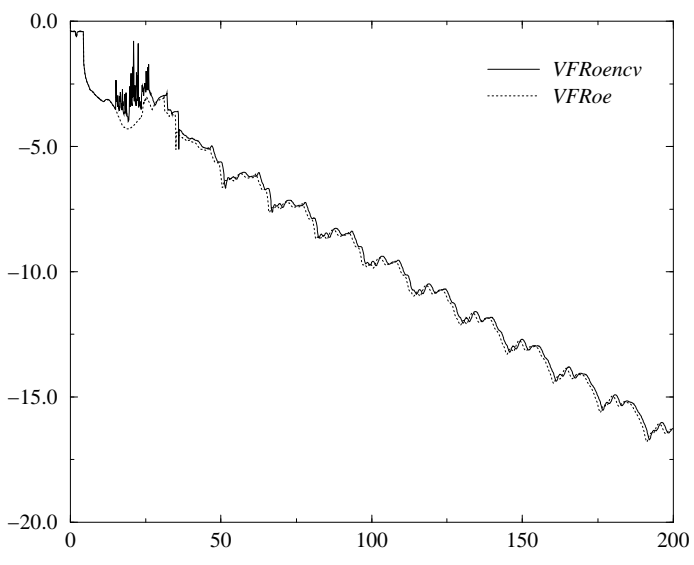

Figure 28: Subcritical flow : normalised time variation in $L^{2}$-norm

$Q(t=0, x)=0 \mathrm{~m}^{2} / \mathrm{s}$ and $h(t=0, x)=2 \mathrm{~m}$. The mesh is composed of 300 cells and the CFL 
number is 0,4 . Both methods provide profiles of water height which are very close to each other in

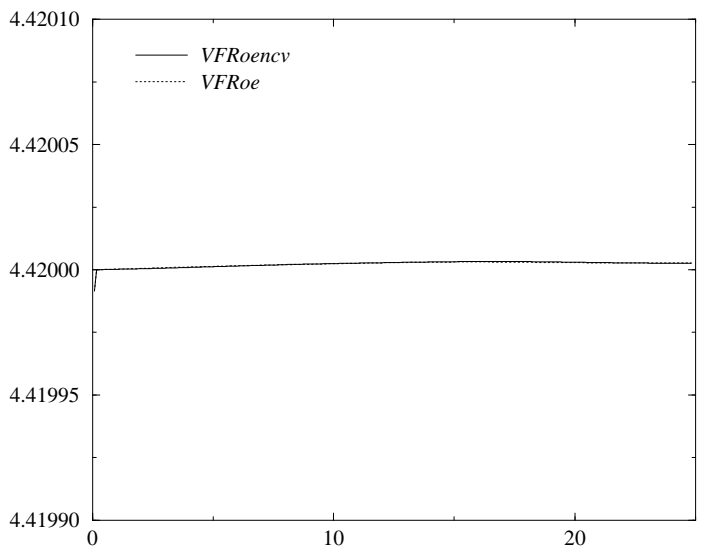

Figure 29: Subcritical flow : discharge

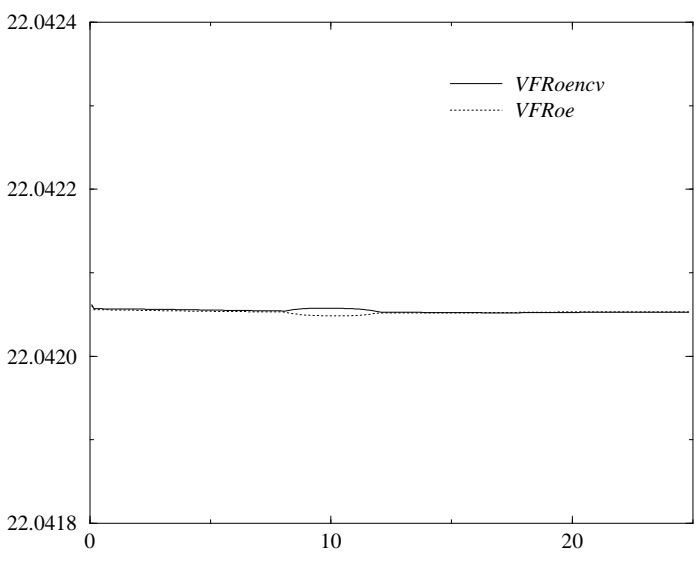

Figure 30: Subcritical flow : $\psi$

figure 27. Moreover, the time variation decreases with the same slope on figure 28. Figures 29 and 30 show that the VFRoe scheme and the VFRoencv scheme both compute almost constant values of $Q$ and $\psi$, in agreement with the analytic solution (the relative error in $L^{\infty}$-norm is around $10^{5}$. Thus, the VFRoe scheme provide too accurate results on this test case. As the high-resolution Godunov method proposed by R.J. LeVeque in [20], the VFRoe scheme fails to deal with occurence of transcritical flow by an inlet condition (see test 6.3), even with the higher order extension.

\section{References}

[1] C. Bon, Modélisation et simulations numériques d'écoulements hydrauliques et de ruissellement en topographie quelconque, PhD thesis, Université Bordeaux I, France, 1997.

[2] R. Botchorishvili, B. Perthame and A. Vasseur, Schémas d'équilibre pour les lois de conservation scalaires avec des termes sources raides, INRIA Report RR-3891, 2000.

[3] T. Buffard, T. Gallouët and J.M. HÉrard, A naive Godunov scheme to compute a non-conservative hyperbolic system, Int. Series Num. Math., 1998, vol. 129, pp. 129-138.

[4] T. Buffard, T. Gallouët and J.M. Hérard, A naive Godunov scheme to solve shallowwater equations, C. R. Acad. Sci. Paris, 1998, vol. I-326, pp. 885-890.

[5] _ A sequel to a rough Godunov scheme. Application to real gas flows, Computers and Fluids, 2000, vol. 29-7, pp. 813-847.

[6] A. Chinnayya And A.Y. Le Roux, A new general Riemann solver for the shallow-water equations with friction and topography, 1999. Available in the conservation law preprint server http://www. math. ntnu. no/conservation/. 
[7] J.F. Colombenu, Multiplication of distributions, Springer Verlag, 1992.

[8] G. Dal Maso, P.G. Le Floch and F. Murat, Definition and weak stability of non conservative products, J. Math. Pures Appl., 1995, vol. 74, pp. 483-548.

[9] R. Eymard, T. Gallouët and R. Herbin, Finite Volume Methods, In Handbook of Numerical Analysis (Vol. VII), editors : P.G. Ciarlet and J.L. Lions, pp. 729-1020, North-Holland, pp. $729-1020,2000$.

[10] T. Gallouët, J.M. Hérard and N. Seguin, On the use of symmetrizing variables to deal with vacuum, LATP Report 00-32, Université de Provence, France, 2000.

[11] _ Some recent Finite Volume schemes to compute Euler equations using real gas EOS, LATP Report 00-21, Université de Provence, France, submitted for publication, 2000.

[12] P. Garcia-Navarro and M.E. Vazguez-Cendon, On numerical treatment of the source terms in the shallow water equations, Computers and Fluids, 2000, vol. 29-8, pp. 951-979.

[13] S.K. Godunov, A difference method for numerical calculation of discontinous equations of hydrodynamics, Mat. Sb., 1959, pp. 271-300. In Russian.

[14] L. Gosse and A.Y. Le Roux, A well balanced scheme designed for inhomogeneous scalar conservation laws, C. R. Acad. Sci. Paris, 1996, vol. I-323, pp. 543-546.

[15] N. Goutal and F. Maurel, Proceedings of the 2nd workshop on dam-break wave simulation, EDF-DER Report HE-43/97/016/B, 1997.

[16] J.M. Greenberg and A.Y. Le Roux, A well balanced scheme for the numerical processing of source terms in hyperbolic equation, SIAM J. Numer. Anal., 1996, vol. 33-1, pp. 1-16.

[17] J.M. Greenberg, A.Y. Le Roux, R. Baraille and A. Noussair, Analysis and approximation of conservation laws with source terms, SIAM J. Numer. Anal., 1997, vol. 34-5, pp. 1980-2007.

[18] A.Y. Le Roux, Discrétisation des termes sources raides dans les problèmes hyperboliques, In Systèmes hyperboliques: Nouveaux schémas et nouvelles applications. Écoles CEA-EDFINRIA "problèmes non linéaires appliqués", INRIA Rocquencourt (France), March 1998. In French.

[19] R..J. LeVeque, Numerical Methods for Conservation Laws, Birkhäuser-Verlag, Basel, 1990.

[20] — Balancing source terms and flux gradients in high-resolution Godunov methods, $J$. Comp. Phys, 1998, vol. 146, pp. 346-365.

[21] P.L. Lions and B. Mercier, SiAM J. Numer. Anal., 1979, vol. 16-6, pp. 964-979.

[22] J.M. Masella, I. Faille and T. Gallouët, On an approximate Godunov scheme, Int. J. of Comp. Fluid Dyn., 1999, vol. 12, pp. 133-149.

[23] P.L. Roe, Approximate Riemann solvers, parameter vectors and difference schemes, J. Comp. Phys, 1981, vol. 43, pp. 357-372. 
[24] E.F. Toro, Riemann solvers and numerical methods for fluid dynamics, Springer Verlag, 1997.

[25] B. Van Leer, Toward the ultimate conservative difference scheme V. A second order sequel to Godunov's method, J. Comp. Phys, 1979, vol. 32, p. 101.

[26] N.N. Yanenko, Méthode à pas fractionnaires. Résolution de problèmes polydimensionnels de physique mathématique, Librairie Armand Colin, 1968. Traduit du russe par P.A. Nepomiastchy. 